\title{
Cpk2, a Catalytic Subunit of Cyclic AMP-PKA, Regulates Growth and Pathogenesis in Rice Blast
}

\author{
Poonguzhali Selvaraj ${ }^{1 *}$, Qing Shen ${ }^{1 \dagger}$, Fan Yang ${ }^{1}$ and Naweed I. Naqvi ${ }^{1,2 *}$ \\ 1 Temasek Life Sciences Laboratory and Department of Biological Sciences, 1 Research Link, National University of \\ Singapore, Singapore, Singapore, ${ }^{2}$ School of Biological Sciences, Nanyang Technological University, Singapore, Singapore
}

\section{OPEN ACCESS}

Edited by:

Charley Christian Staats, Federal University of Rio Grande do

Sul (UFRGS), Brazil

Reviewed by:

Zonghua Wang,

Fujian Agriculture and Forestry University, China

Shihua Wang,

Fujian Agriculture and Forestry University, China

*Correspondence: Naweed I. Naqvi

naweed@ttl.org.sg

Poonguzhali Selvaraj guzhali@tll.org.sg

tThese authors have contributed equally to this work.

Specialty section: This article was submitted to Fungi and Their Interactions, a section of the journal

Frontiers in Microbiology

Received: 30 August 2017 Accepted: 06 November 2017 Published: 21 November 2017

Citation:

Selvaraj P, Shen Q, Yang Fand Naqvi NI (2017) Cpk2, a Catalytic

Subunit of Cyclic AMP-PKA, Regulates Growth and Pathogenesis in Rice Blast.

Front. Microbiol. 8:2289. doi: 10.3389/fmicb.2017.02289
The cAMP-Protein Kinase A signaling, anchored on CpkA, is necessary for appressorium development and host penetration, but indispensable for infectious growth in Magnaporthe oryzae. In this study, we identified and characterized the gene encoding the second catalytic subunit, CPK2, whose expression was found to be lower compared to CPKA at various stages of pathogenic growth in $M$. oryzae. Deletion of CPK2 caused no alterations in vegetative growth, conidiation, appressorium formation, or pathogenicity. Surprisingly, the cpkA $\Delta c p k 2 \Delta$ double deletion strain displayed significant reduction in growth rate and conidiation compared to the single deletion mutants. Interestingly, loss of CPKA and CPK2 resulted in morphogenetic defects in germ tubes (with curled/wavy and serpentine growth pattern) on hydrophobic surfaces, and a complete failure to produce appressoria therein, thus suggesting an important role for CPK2-mediated CAMP-PKA in surface sensing and response pathway. CPKA promoter-driven expression of CPK2 partially suppressed the defects in host penetration and pathogenicity in the $c p k A \triangle$. Such ectopic CPK2 expressing strain successfully penetrated the rice leaves, but was unable to produce proper secondary invasive hyphae, thus underscoring the importance of CpkA in growth and differentiation in planta. The Cpk2-GFP localized to the nuclei and cytoplasmic vesicles in conidia and germ tubes. The Cpk2-GFP colocalized with CpkA-mCherry on vesicles in the cytosol, but such overlap was not evident in the nuclei. Our studies indicate that CpkA and Cpk2 share overlapping functions, but also play distinct roles during pathogenesis-associated signaling and morphogenesis in the rice blast fungus.

Keywords: Magnaporthe, rice blast, cyclic AMP, protein kinase A, catalytic subunit, localization

\section{INTRODUCTION}

The Protein kinase A (PKA) family of Ser/Thr kinases is highly conserved in eukaryotes, and serves important phosphorylation-dependent functions in signal transduction and development (Hanks and Hunter, 1995). The PKA holoenzyme is an inactive heterotetramer composed of two regulatory (R) and two catalytic (C) subunits and the cooperative binding of two cAMP molecules to the R subunit frees and activates the $\mathrm{C}$ subunits to phosphorylate hundreds of targets and regulate a vast swath of biochemical and metabolic processes.

The catalytic subunit of PKA (PKA-C) is a typical structure for protein kinases and PKA signaling plays a central role in vegetative growth, development, mating, stress response, and 
pathogenicity in various fungi (Lengeler et al., 2000; D'Souza and Heitman, 2001). Multiple PKA isoforms are expressed in mammalian cells and have tissue-specific roles indicative of functional diversity. Three TPK genes encoding PKA-C were identified in the budding yeast Saccharomyces cerevisiae, and subsequently shown to share redundant and distinct functions in viability and in pseudohyphal morphogenesis, respectively (Toda et al., 1987; Robertson and Fink, 1998; Pan and Heitman, 1999). In Candida albicans, the catalytic isoforms Tpk1p and Tpk $2 \mathrm{p}$ share positive roles in cell growth, while they have distinct roles in hyphal morphogenesis, stress response and regulation of glycogen metabolism (Sonneborn et al., 2000; Bockmühl et al., 2001; Cloutier et al., 2003). Meanwhile, numerous known filamentous fungi are found to possess only two distantly related PKA-C isoforms with varied functions (Lee et al., 2003; Banno et al., 2005; Ni et al., 2005; Schumacher et al., 2008). In most plant pathogenic fungi, deletion of one PKA-C isoform resulted in profound effects in virulence. For instance, of the two PKA catalytic subunits, only Adr1 kinase activity is essential for the dimorphic transition and pathogenesis in Ustilago maydis (Dürrenberger et al., 1998). Similarly, only one PKA isoform plays a predominant role in other phytopathogens like Colletotrichum trifolii, C. lagenarium, Botrytis cinerea and Setosphaeria turcica that utilize appressoria to penetrate and infect the host; or in Mycosphaerella graminicola and Verticillium dahlia, that invade the host through stomata or other natural openings (Yang and Dickman, 1999; Takano et al., 2001; Yamauchi et al., 2004; Mehrabi and Kema, 2006; Schumacher et al., 2008; Tzima et al., 2010; Hao et al., 2015).

The Rice Blast pathosystem has been extensively analyzed at the molecular level, and serves as a model in the study of plant-fungus interactions (Liu et al., 2013), and in tackling global food security (Nalley et al., 2016). The cAMP/PKA signaling in $M$. oryzae plays an important role in surface sensing, appressorium morphogenesis, turgor generation, and in regulating plant infection ( $\mathrm{Li}$ et al., 2012; Yan and Talbot, 2016). Different components of the G-protein signaling such as the G $\alpha$, MagA, MagB or MagC, two G $\beta$ (Mgb1 and Mgb2), a $\mathrm{G} \gamma$ subunit, and the Rgs1 (regulator of G-protein signaling 1) have been characterized (Liu and Dean, 1997; Fang and Dean, 2000; Nishimura et al., 2003; Dean et al., 2005; Liu et al., 2007; Ramanujam et al., 2012). Anchoring and trafficking of G-protein signaling components on late endosomes endows $M$. oryzae with the ability to specifically activate, integrate and achieve modularity and spatio-temporal control of signaling responses critical for pathogenesis (Ramanujam et al., 2013). Downstream of the $G$ proteins, the adenylate cyclase Mac1 (that synthesizes cAMP), its suppressor Sum1, and the cAMP phosphodiesterases have been characterized too (Choi and Dean, 1997; Adachi and Hamer, 1998; Ramanujam and Naqvi, 2010; Zhang et al., 2011). Mutants disrupted in the catalytic subunit gene CPKA exhibit normal growth and conidiation, but show delayed appressorium formation and loss of pathogenicity, which results from the defects in appressorial function (Mitchell and Dean, 1995; Xu et al., 1997). We showed that loss of the regulatory subunit of PKA $(R P K A)$ results in complete loss of pathogenicity; and a suppressor mutant that partially restores the pathogenicity in
rpkA $\Delta$ represents a point mutation in the CPKA locus (Selvaraj et al., 2017). Recently, the second catalytic subunit of PKA, Cpk2, has been characterized in M. oryzae (Li Y. et al., 2017). These studies confirm a crucial role for cAMP/PKA signaling in the development and pathogenicity of $M$. oryzae.

In this study, we set out to investigate the role of CPK2 through gene-deletion analysis and functional characterization. To gain further insights into the function of cAMP-PKA signaling in $M$. oryzae pathogenicity, we also created a $c p k A c p k 2$ double deletion mutant and also analyzed the subcellular localization of Cpk2. We show that the Cpk2 activity is largely redundant with CpkA, and that both the catalytic subunits act in concert to regulate hyphal growth and play overlapping roles in conidiation and appressorium formation in $M$. oryzae. Importantly, these processes are dependent on Cpk2, since CPK2 deletion removes even the residual virulence associated with loss of CPKA. The expression of CPK2 under the CPKA promoter, or the swapping in of $C P K 2$ coding region for $C P K A$, restored the pathogenicity in $M$. oryzae cpkA null mutant. Unlike CpkA, the Cpk2 subunit localized predominantly to the nucleus in rice blast. Taken together, this study underscores the importance of cyclic AMP PKA signaling in the pathogenic differentiation of M. oryzae.

\section{EXPERIMENTAL PROCEDURES}

\section{Strains, Growth Conditions, and Transformation}

The Magnaporthe oryzae strain B157 (wild type - WT) obtained from the Directorate of Rice Research (Hyderabad, India) and its transformants/derived strains were routinely cultured on prune agar medium (PA) at $28^{\circ} \mathrm{C}$ for $7-10$ days. Preparations of the media, assessment of growth, conidiation and appressorium formation and Agrobacterium tumefaciensmediated transformation (AtMT) were carried out as routinely (Ramanujam and Naqvi, 2010; Deng et al., 2012). Requisite transformants were screened by Southern blot analysis and/or locus-specific PCR and in each case, two confirmed strains were selected for further observations.

\section{Nucleic Acid Manipulation and Sequence Analysis}

The CPK 2 orthologs were identified by searching the Genbank and fungal genome databases using the BLAST program (Altschul et al., 1997) and multiple sequence alignments were carried out with ClustalW (Thompson et al., 1994) and Boxshade ${ }^{1}$. Plasmid DNA extractions and genomic DNA extraction from the complete medium (CM) grown mycelium were carried out using standard kits; Geneaid High Speed Plasmid Mini kit and Yeast DNA purification kit (Epicenter Biotechnologies, United States) according to the protocols mentioned therein. The PCR primers used in this study are mentioned in Supplementary Table S1. Nucleotide sequencing was performed using the ABI Prism big dye terminator method

\footnotetext{
${ }^{1}$ http://www.ch.embnet.org/software/BOX_form.html
} 
(PE Applied Biosystems). Southern blot analysis was performed by using the Enhanced chemiluminescent labeling and detection kit (Amersham Biosciences, RPN2108). Standard procedures were adopted for DNA restriction, agarose gel transformation and hybridizations for Southern blot (Sambrook et al., 1989).

\section{Generation of cpkA and cpk2 Deletion Mutants, Overexpression Strains and GFP Fusion Constructs}

To generate deletion mutants of $c p k \mathrm{~A}$ and $c p k 2$, gene replacement vectors encoding glufosinate ammonium resistance in pFGL97 or the hygromycin resistance in pFGL44 flanking the respective ORF were constructed using ligation PCR approach and then transformed to WT using AtMT. To get $c p k A \Delta c p k 2 \Delta$, the $c p k 2 \Delta$ construct was introduced into $c p k \mathrm{~A} \Delta$ strain. The CPK2-GFP in pFGL820 (encoding sulfonyl urea resistance gene cassette) was constructed by sequential cloning of the eGFP ORF, the last $1 \mathrm{~kb}$ and the downstream fragment of CPK2 ORF to yield the final construct pCPK2-GFP-Trpc construct (Selvaraj et al., 2017). The GFP-CPK2 overexpression construct was created by fusing the Moh3 promoter with the cpk2 ORF, sequentially cloned in to pFGL1010G which encodes sulfonyl urea resistance with an ilV locus which facilitate ectopic single copy integration (Yang and Naqvi, 2014). To construct a cpk2 ORF overlapping cpkA vector, the ORF of cpk2 was fused with the promoter of cpkA and ligated to pFGL880 (encoding sulfonyl urea resistance gene cassette) which already contained the CPKA $3^{\prime}$ UTR.

\section{Protein Isolation and Western Blot Analysis}

Total proteins $(\sim 30 \mu \mathrm{g})$ from mycelia collected from 2 days old CM cultures extracted were separated on a 10\% SDSPAGE gel and transferred to PVDF membranes for western blot analysis as described (Bruno et al., 2004; Liu et al., 2011). TEY phosphorylation of MAPKs was detected with the PhophoPlus p44/42 MAPK antibody kit (Cell Signaling Technology, Beverly, MA, United States) according to the manufacturer's instructions. A monoclonal anti-actin antibody (Abcam) was used to detect actin.

\section{Plant Cultivar, Growth and Blast Infection Assays}

Rice cultivar CO39 and barley cultivar Express susceptible to M. oryzae strain B157 were used for blast infection assays. Rice was grown at $80 \%$ humidity at $28^{\circ} \mathrm{C}$ and Barley was grown at $60 \%$ humidity at $24^{\circ} \mathrm{C}$ (day) and $22^{\circ} \mathrm{C}$ (night) with $12 \mathrm{~h}: 12 \mathrm{~h}$ day:night cycles in a growth chamber. For plant infection assays, freshly harvested conidia at a concentration of $1 \times 10^{6} / \mathrm{ml}$ in $0.2 \%$ gelatin were used. Plant inoculation, incubation and lesion examination were conducted as mentioned previously (Ramanujam and Naqvi, 2010; Bosch et al., 2012; Selvaraj et al., 2017). Rice leaf sheath infection assay was performed as described (Kankanala et al., 2007). Surface-sterilized rice seeds germinated and grown in direct contact with the fungal mycelial plugs were examined for black or browning lesions in the roots after 2 weeks to assess the root infection (Dufresne and Osbourn, 2001).

\section{Real Time qRT-PCR Analysis}

Total RNA isolation from the mycelia or the frozen germlings at different time points and plant samples was carried out using RNeasy Plant Mini kit (QIAGEN, United States). The first strand cDNA synthesis and qRT-PCR were performed as mentioned previously (Patkar et al., 2012). All qRT-PCR reactions were conducted twice with three replications for each sample using the requisite primer sets for open reading frames for $c p k \mathrm{~A}, c p k 2$ and $\beta$-tubulin (TUB2) mentioned (Supplementary Table S1). The abundance of the gene transcripts was calculated by the $2^{-\Delta \Delta C_{\mathrm{T}}}$ method with $\beta$-tubulin as the internal control.

\section{Assays for cAMP-Dependent Protein Kinase A (PKA) and Quantification of Intracellular CAMP}

PKA assay was performed using a non-radioactive cAMPdependent protein kinase assay system fluorescent using the PKA model substrate, Kemptide (Promega, Madison, WI, United States), following the manufacturer's instructions and the sample preparation, determination of protein concentration were carried out were prepared as mentioned previously (Kang et al., 1999; Selvaraj et al., 2017). The samples for cAMP estimation were prepared as described previously (Liu et al., 2007; Ramanujam and Naqvi, 2010), and the assays were carried out using the CAMP Biotrak Immuno-assay system (Amersham Biosciences, Piscataway, NJ, United States) according to the manufacturer's protocol.

\section{Microscopy, Image Analysis and Processing}

Staining with DAPI (diamidino-2-phenylindole; Sigma-Aldrich, United States) was carried out essentially as described already (Patkar et al., 2010; Ramanujam and Naqvi, 2010). Bright field and epifluorescence microscopy was performed with an Olympus IX71 or BX51 microscope (Olympus, Tokyo, Japan) using a Plan APO 100X/1.45 or UPlan FLN 60X/1.25 objective and appropriate filter sets. Images were captured with Photometrics CoolSNAP HQ camera (Tucson, AZ, United States) and processed using MetaVue (Universal Imaging, Downingtown, PA, United States), and Adobe Photoshop 7.0.1 (Mountain View, CA, United States). Time-lapse or live cell fluorescence microscopy was performed using a an UltraView RS-3 spinning disk confocal system (PerkinElmer Inc., United States) using a $491 \mathrm{~nm} 100 \mathrm{~mW}$ and a $561 \mathrm{~nm} 50 \mathrm{~mW}$ laser illumination under the control of MetaMorph Premier Software (Ramanujam et al., 2013; Selvaraj et al., 2017). Typically, z-stacks consisted of $0.5 \mu \mathrm{m}$-spaced planes for every time point. Image processing and preparation was performed using $\mathrm{Fiji}^{2}$, and Adobe Photoshop.

\section{Statistical Analyses}

The one-way analysis of variance (ANOVA) tests were performed in order to assess whether the differences between the average responses of the treatments were significant. $P$ values lower than 0.05 were considered to be significant. Statistical data was

${ }^{2}$ http://fiji.sc/wiki/index.php/Fiji 
analyzed by the Student's $t$-test to ascertain the significance of individual treatments and replicates, wherever applicable.

\section{RESULTS}

\section{Identification and Gene-Deletion Analysis of CPK2 in M. oryzae}

Analysis of the genome sequence of $M$. oryzae $e^{3}$ revealed an open reading frame (ORF) that encodes a catalytic subunit of PKA, CPK2 (MGG_02832; contig 6-2325: coordinates 2566-1350), which was distinct from the CPKA locus. CPK2 encodes a member of the Class II PKA subunits unique to filamentous fungi (Schumacher et al., 2008). MGG_02832 (GI: 2682385) showed the presence of three exons spanning a $1474 \mathrm{bp}$ ORF, predicted to encode a 408-amino acid polypeptide (XP_003720907.1). The Cpk2 protein contains a typical serine/threonine kinase domain as well as a C-terminal AGC domain, which is representative of a large family of kinases and is conserved in numerous PKA catalytic subunits (Pearce et al., 2010). M. oryzae Cpk2 shows 40-53\% amino acid identity to Class II PKAs in yeast (Toda et al., 1987), A. fumigatus (Liebmann et al., 2004), B. cinerea (Schumacher et al., 2008), and U. maydis (Dürrenberger et al., 1998). A detailed phylogenetic analysis of the class II PKAs, including the $M$. oryzae $\mathrm{Cpk} 2$, has been described previously (Schumacher et al., 2008). The CpkA and Cpk2 proteins of M. oryzae share approximately $48 \%$ sequence identity, with the highest degree of divergence within the $\mathrm{N}$-terminal region. The highly conserved protein kinase domain of Cpk2 extends from 75 and 350 aa and the nucleotide binding site LGTGFARV (81-89 aa) differed by single aa from the conserved motif within CpkA, whereas the active catalytic domain RDLKPEN (203-215 aa) was identical (Supplementary Figure S1).

We carried out gene-deletion analyses to determine the relative contributions of each catalytic subunit of PKA, CPKA and $C P K 2$, to the pathogenicity of $M$. oryzae. In addition, a strain deficient for both CPKA and CPK2 was generated illustrating that cAMP-PKA signaling is not essential for viability in M. oryzae. The transformants were confirmed through Southern blotting and locus specific PCR (Supplementary Figure S2), and in all cases at least two independent strains were characterized in detail.

\section{Cpk2 and CpkA Are Required for Vegetative Growth and Conidiation in \\ M. oryzae}

The colony morphology and the radial growth of the individual $c p k \mathrm{~A} \Delta$ and $c p k 2 \Delta$ strains were indistinguishable from the WT, while the $c p k A \Delta c p k 2 \Delta$ showed reduced radial growth producing small colonies with fluffy aerial growth (Figures 1A,C). Although CPKA is dispensable for vegetative growth and conidiation, it regulates appressorium formation and function, with the cpkA $\Delta$ strain displaying long germ tubes and delayed appressorium formation (Mitchell and Dean, 1995; Xu et al., 1997). No apparent changes were observed in conidiation in $\operatorname{cpkA} \Delta$ and $c p k 2 \Delta$

${ }^{3} \mathrm{http} / /$ fungi.ensembl.org/Magnaporthe_oryzae/Gene/Summary compared to the WT. Based on the cpk $2 \Delta$ phenotypes, we inferred that Cpk2 functionality could be elucidated better in the context of the loss of CpkA activity. Accordingly, the deletion of $c p k 2$ in $c p k \mathrm{~A} \Delta,(c p k \mathrm{~A} \Delta c p k 2 \Delta)$, led to further delay and reduction in conidiation compared to the WT or the individual mutants. The WT, $c p k A \Delta$ or $c p k 2 \Delta$ strains produced conidia within $24 \mathrm{~h}$ of light exposure, whereas the $c p k \mathrm{~A} \Delta c p k 2 \Delta$ mutant did not initiate conidia formation even after $48 \mathrm{hpi}$. At 7-10 days of exposure to light, the double mutant produced about 10-fold lesser $(p<0.01)$ conidia than the WT (Figures 1B,C). However, the conidia produced were three celled and with no apparent abnormalities in shape or size, thus implying that complete loss of PKA activity would adversely affect asexual development. We conclude that cAMP-PKA activity is essential for conidiation in M. oryzae; and that CpkA and Cpk2 possess overlapping roles in regulating such metabolic activation and initiation of asexual reproduction therein.

\section{CPK2 Plays a Significant Role in Appressorium Formation in $M$. oryzae}

The cpkA $\Delta$ showed a significant delay in appressoria formation on hydrophobic/inductive surfaces, and the appressoria produced were smaller with long germ tubes. The cpk2 $\Delta$ produced normal appressoria indistinguishable from WT. Interestingly, the $c p k \mathrm{~A} \Delta c p k 2 \Delta$ conidia germinated normally, but failed to elaborate appressoria on inductive surfaces even with excess cAMP. Germ tubes produced by $c p k A \Delta c p k 2 \Delta$ conidia were very long, curled, showed periodic clockwise twists, and did not produce appressoria even at $32 \mathrm{hpi}$ (Figures 2A-C). Unlike the WT, $c p k \mathrm{~A} \Delta$ or $c p k 2 \Delta$ that elaborate appressoria on non-inductive surfaces in response to exogenous cAMP, the $c p k \mathrm{~A} \Delta c p k 2 \Delta$ was non-responsive to the cAMP stimulus and failed to elaborate appressoria (Figure 2D). Appressorium morphogenesis is tightly regulated by the cell cycle, with DNA replication and one round of mitosis being essential for the initiation of appressoria in M. oryzae (Veneault-Fourrey et al., 2006; Saunders et al., 2010; Li C. et al., 2017). We inferred that the defect in appressorium formation in the $c p k A \Delta c p k 2 \Delta$ is likely due to the inability to sense and/or respond to cAMP in addition to defects in surface sensing and adhesion. The delayed appressorium formation in $c p k A \Delta$, and the inability of the $c p k A \Delta c p k 2 \Delta$ to initiate appressoria, further underscore the importance of CPK2 in surface sensing and appressorium formation in M. oryzae.

To further clarify the role of $C P K 2$, we added the cAMPPKA inhibitor KT5720 to the conidia on coverslips and checked appressorium formation at $24 \mathrm{~h}$. Addition of KT5720 delayed appressorium formation in the WT, but had no effect on appressorium morphology. KT5720 showed dose-dependent reduction in appressorium formation ability in $c p k A \Delta$ or $c p k 2 \Delta$ conidia. At $2 \mu \mathrm{M}$ KT5720, appressorium formation decreased from 80 to $42 \%$ in the $c p k A \Delta(p=0.004)$ compared to the solvent control, whereas the reduction was from 93 to $79 \%(p=0.009)$ in $c p k 2 \Delta$. At higher concentration $(5 \mu \mathrm{M})$, the effect of KT5720 was highly variable and pleiotropic, with the resultant appressoria being malformed and/or underdeveloped; and the treated conidia 
A

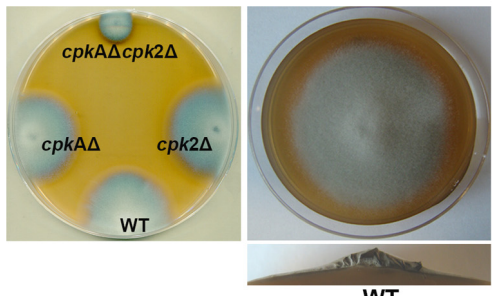

WT

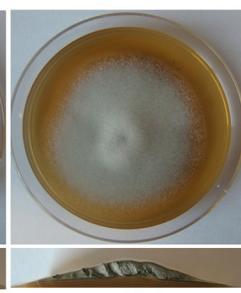

cpkA $\Delta$

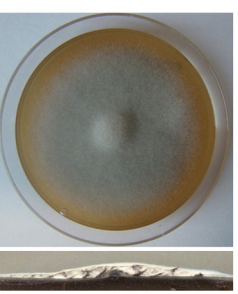

$c p k 2 \Delta$

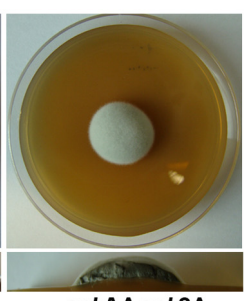

cpkA $\Delta c p k 2 \Delta$
B

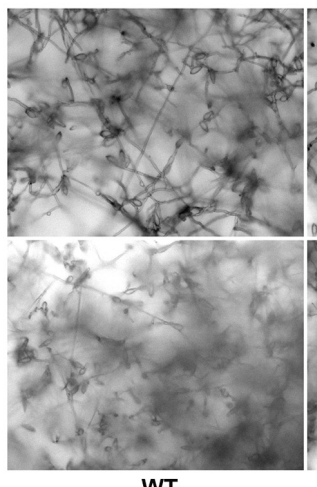

WT

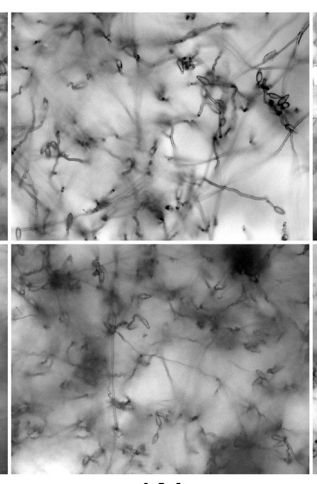

cpkA $\Delta$

C

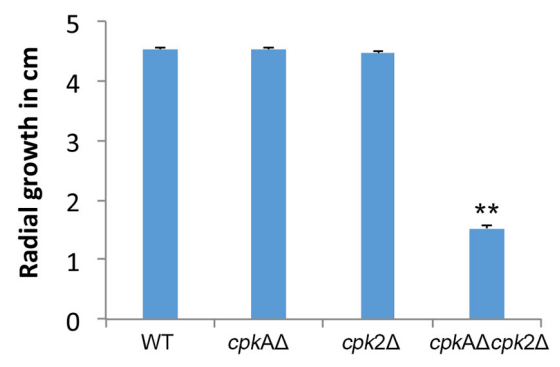

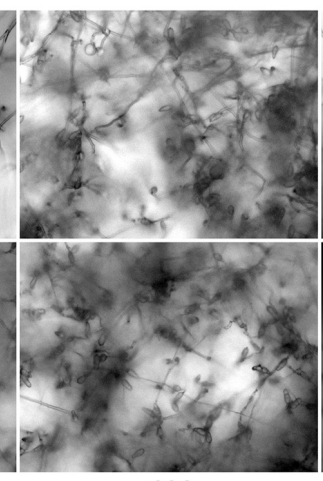

$c p k 2 \Delta$

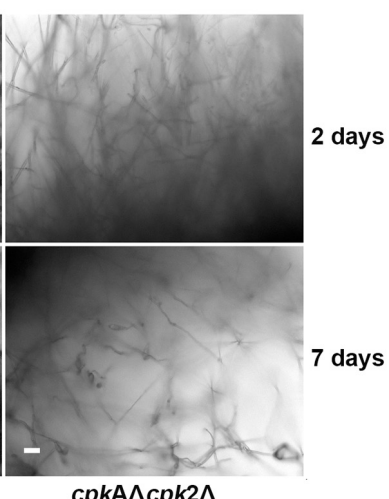

$c p k A \Delta c p k 2 \Delta$

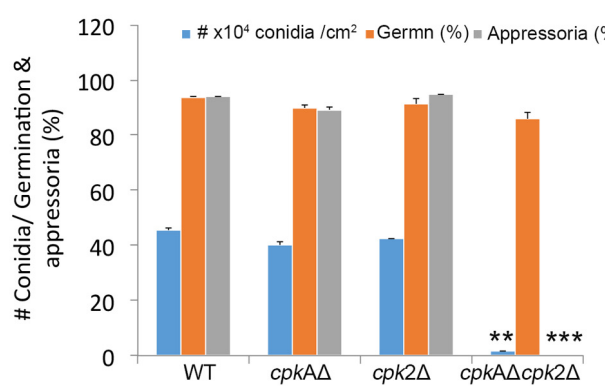

FIGURE 1 | Cpk2-mediated CAMP-PKA signaling is necessary for proper vegetative and asexual development in M. oryzae (A) Radial and aerial hyphal growth of the wild type (WT) and the indicated PKA-C mutant strains. Mycelial plugs inoculated on PA medium was cultured in the dark at $28^{\circ} \mathrm{C}$ for 5 days. The left panel shows the comparative radial growth of individual CPK mutants $c p k A \Delta, c p k 2 \Delta$ and $c p k A \Delta c p k 2 \Delta$ with the WT B157. The radial and cross-sectional view for the aerial hyphal growth of WT and the mutants are shown (right). (B) Bright field micrographs showing the conidiation at $48 \mathrm{~h}$ and 7 days post photoinduction. Individual cpkA $\Delta$ and $c p k 2 \Delta$ produced conidia normally as the WT at $48 \mathrm{~h}$, while $c p k A \Delta c p k 2 \Delta$ showed very few conidia even at 7 dpi. Scale bar $=10 \mu \mathrm{m}$. (C) Bar graphs showing the difference in radial growth (left) and the quantification of conidiation and appressorium formation in WT and PKA-C mutants (right). Values represent mean \pm SE of three independent replicates with approximately 200 conidia assessed per experiment; ${ }^{* *} p<0.001,{ }^{* * *} p<0.0001$

did not show further reduction in the ability to form appressoria. The maximal inhibitor concentration used in our experiment was thus not sufficient to completely block the PKA activity in the WT strain. We conclude that albeit redundant, the second catalytic cAMP-PKA subunit, Cpk2, plays an important role in appressorium formation in the rice blast fungus.

\section{CPKA and CPK2 Are Involved in Regulation of PKA Signaling and Intracellular CAMP Levels}

PKA activity was undetectable in the total protein extracts from the mycelia of $c p k \mathrm{~A} \Delta$ or $c p k \mathrm{~A} \Delta c p k 2 \Delta$, whereas the WT clearly showed the cAMP-dependent PKA activity (Figure 3A). PKA activity could still be detected in the mycelial extracts of $c p k 2 \Delta$ but was very low. The $c p k 2 \Delta$ produced only about 50\% PKA activity in vitro compared to the WT M. oryzae (Figure 3B), indicating that there is no compensatory increase in CpkA activity in the absence of CPK2. However, the function(s) of Cpk2 cannot be ascertained solely on the basis of its enzyme activity; and the regulatory interactions and interdependency between the two isoforms could not be ruled out (Ni et al., 2005). Hence, we carried out qRT-PCR to determine the expression levels of CPKA and CPK2 in order to check if deletion of CPKA affects the expression of $C P K 2$ or vice versa. In WT, albeit having a similar expression pattern, the level of $C P K 2$ was comparatively lower than CPKA at all the time points tested (Figure 3C) indicating that the activity of CpkA alone contributes to the regular functions of cAMP-PKA in the cpk2 $\triangle$ mutant. CPKA and CPK2 were expressed in mycelia and aerial hyphae at comparable levels, and hence the $c p k \mathrm{~A} \Delta c p k 2 \Delta$ was highly impaired in radial and aerial growth. The expression of both isoforms increased in the 

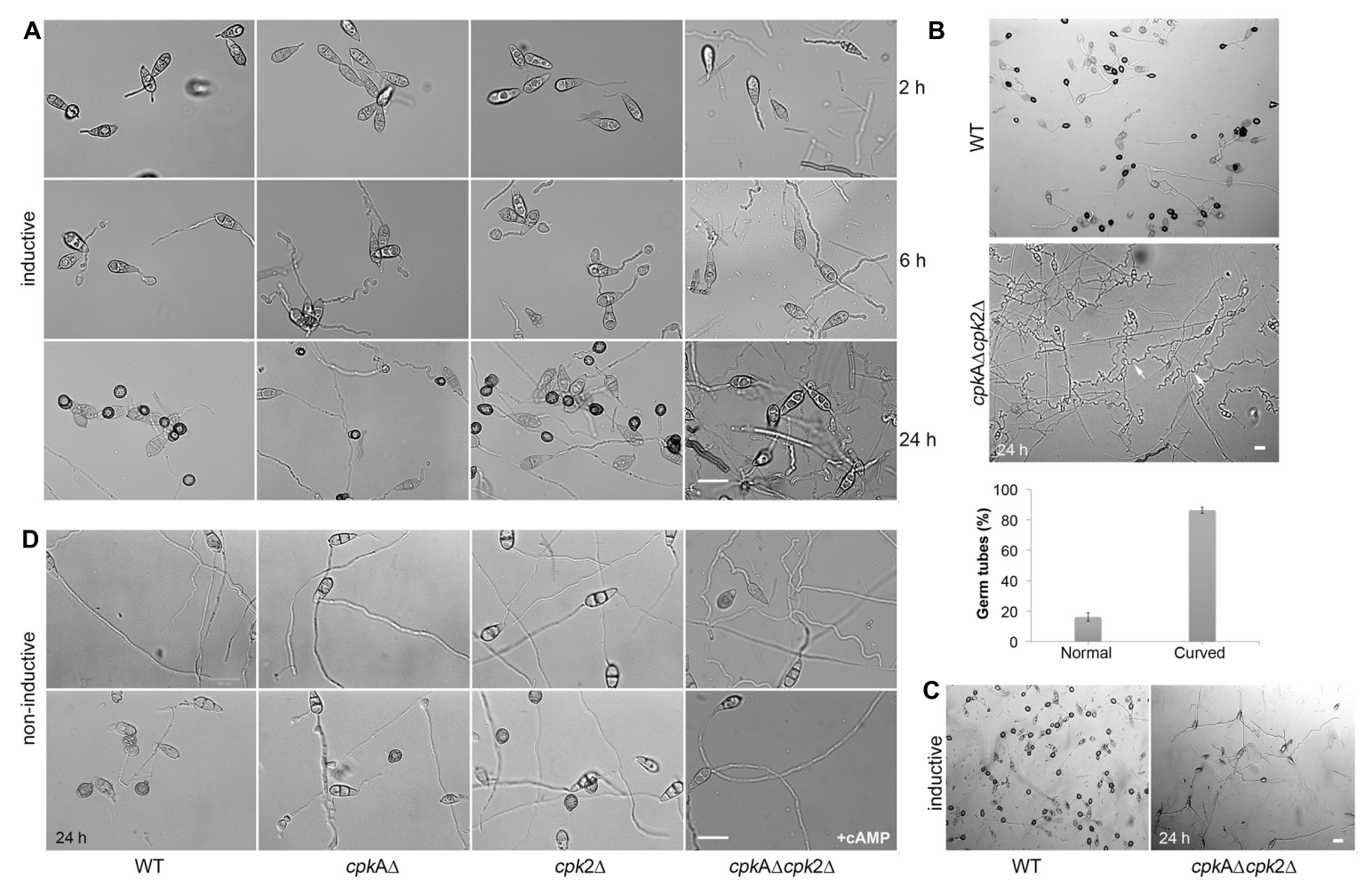

FIGURE 2 | Cpk2 plays a crucial role in appressorium formation. Bright field micrographs of the appressoria formed by WT and CPK mutants on inductive hydrophobic (A) and non-inductive - hydrophilic surfaces (D) at different time points. (A) The cpkA $\Delta c p k 2 \Delta$ did not produce appressoria on inductive surface even at $24 \mathrm{hpi}$, while $c p k A \Delta$ was significantly delayed in appressorium formation. (B) The cpkA $\Delta c p k 2 \Delta$ produced very long, wavy germ tubes without forming appressoria, and the germ tubes showed curled/zig-zag growth unlike the straight/linear growth mode in the WT. Different phenotypes of the germ tube formed by cpkA $\Delta c p k 2 \Delta$ were quantified and represented as a bar graph. (C) Addition of exogenous CAMP (10 mM) to the conidia suppressed the wavy growth pattern, but failed to restore the appressoria formation in the $c p k A \Delta c p k 2 \Delta$. (D) Addition of exogenous cAMP (10 mM) to the conidia restored the appressorium formation in WT and $c p k A \Delta$, $c p k 2 \Delta$ on non-inductive surface while cpkA $\Delta c p k 2 \Delta$ was non-responsive to such exogenous cAMP. Scale bar $=10 \mu \mathrm{m}$.

appressoria at $8 \mathrm{~h}$, which could be responsible for the indicated high levels of PKA activity during appressorium formation (Kang et al., 1999). Based on the higher levels of transcription of the PKA isoforms in conidia and appressoria, we infer their functional importance in conidia and appressoria formation and pathogenicity. In comparison to the WT, the expression level of $C P K 2$ in cpkA $\triangle$ did not show any change at any of the time points analyzed (Figure 3D). Similarly, the level of CPKA transcription was comparable in the cpk2 $\Delta$ and the WT during the pathogenic phase (Figure 3E). The expression levels were comparatively higher in the aerial hyphae and conidia compared to the other phases of growth or pathogenesis. However, the overall increase was less than 2 fold, and therefore considered insignificant. Thus, we conclude that deletion of CPKA or CPK2 had only a minor effect on the transcript levels of the other PKA isoform, thus ruling out the possibility of co-transcriptional regulation between CPKA and CPK2 in M. oryzae.

Compared to the WT, the PKA-C mutants showed increased intracellular accumulation of cAMP. The cpkA $\Delta$ showed higher cAMP levels than $c p k 2 \Delta$, consistent with the predominant role for CpkA in overall PKA activity/function. However, $c p k 2$ deletion either in WT or $c p k A \Delta$ led to an increase in overall cAMP concentration indicating that $\mathrm{Cpk} 2$ also acts as a cAMP effector in M. oryzae. The cAMP increased to very high levels in $c p k A \Delta c p k 2 \Delta$ (about 30-fold increase) indicating an additive effect of the loss of both catalytic subunits of cAMP-PKA (Figure 4A). We infer that cAMP-PKA activity limits the intrinsic cAMP to a threshold/moderate level to maintain normal cellular functions; or conversely, the activation of PKA dampens the intracellular cAMP pool. We conclude that $\mathrm{Cpk} 2$ acts in concert with CpkA to regulate the overall accumulation and dynamics of cAMP signaling in M. oryzae.

\section{Pmk1 MAPK Phosphorylation Is Affected in the cAMP-PKA Mutants}

The non-responsiveness for cAMP in $c p k A \Delta c p k 2 \Delta$ that resembles the pmk1 $\Delta$ phenotype (Xu and Hamer, 1996; Kou et al., 2016) led us to assess whether the Pmk1 MAPK activation is compromised in the PKA-C mutants. Therein, we assayed the phosphorylation of Pmk1 with the anti-TpEY specific antibody that detects the phosphorylation of both Pmk1 and Mps1 MAPKs (Zhao et al., 2005). In WT, a band of 42 and $46 \mathrm{kDa}$ indicating the phosphorylation of Pmk1 and Mps1 were observed, while in pmk1 $\Delta$ only the Mps1 phosphorylation was evident (Figure 4B). A relatively weak phosphorylation 


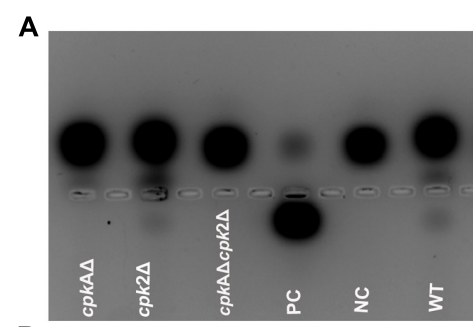

B

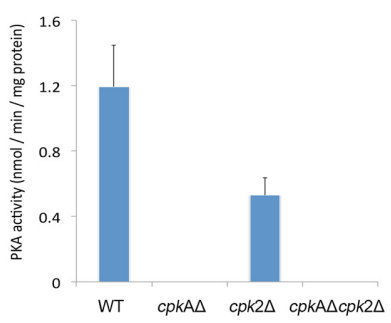

WT $\quad$ cpkA $\triangle \quad c p k 2 \triangle \quad c p k A \triangle c p k 2 \triangle$
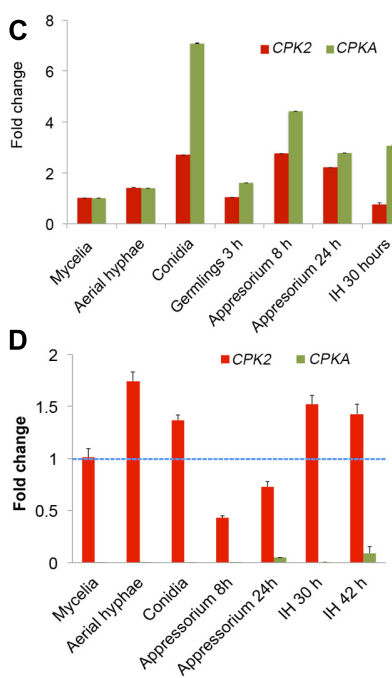

2
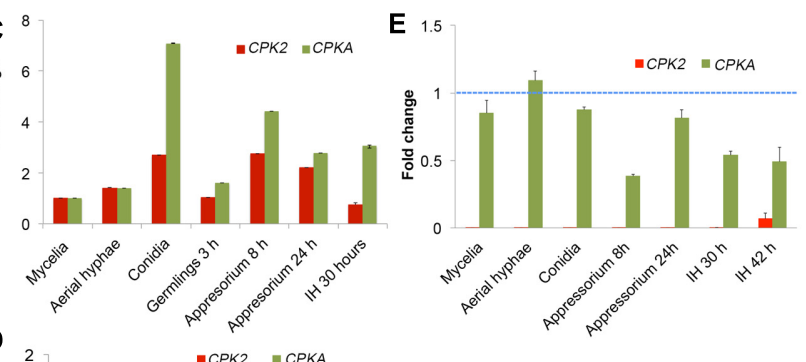

FIGURE 3 | Catalytic activity and transcriptional regulation of the PKA-C subunits in M. oryzae. (A) PKA enzyme activity was monitored by gel electrophoresis showing the migration of phosphorylated substrate toward the anode. PC-positive control; NC-negative control. PKA activity was analyzed in the total protein extracts from the frozen mycelia of WT and the indicated CPK mutants. The results were consistent with repeated experiments. (B) Bar graph showing the PKA activity in WT and the listed PKA-C mutants. Each value represents a mean \pm SE of three replications. Graphical representation of the fold change in the transcript/mRNA levels of CPKA and CPK2 in WT (C), cpkA $\triangle$ (D) and cpk2 $\Delta$ (E) at different stages of vegetative and pathogenic development. Reverse transcriptase RT-PCR was conducted on total RNA extracted from the mycelia and the infection structures mentioned. Fold change in gene expression in WT was calculated from the average of three independent measurements, with $\beta$-Tubulin of $M$. oryzae as internal control and normalized to unit mycelial biomass. For the mutants, for fold change the gene expression was normalized to WT levels (indicated by dotted blue line) at the respective phases with $\beta$-Tubulin of $M$. oryzae as internal control Error bars represent standard error. The experiment was repeated twice with three replicates each.
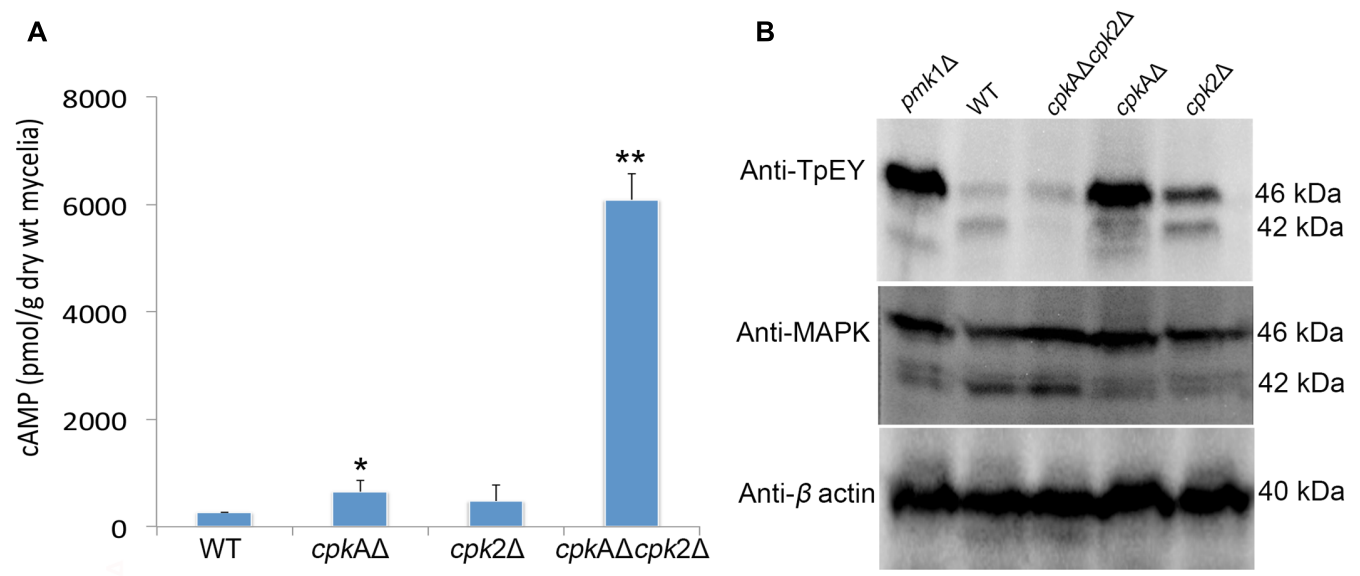

FIGURE 4 | Protein kinase A (PKA) activity regulates the intracellular cAMP levels, and the Pmk1-MAPK signaling in M. oryzae. (A) Quantification of the intracellular cAMP levels in the mycelia of WT and the indicated mutant strains. Values from two biological replicates with two replications for each individual sample were analyzed and the mean value is presented; ${ }^{*} p<0.05$. (B) Western blots showing the phosphorylation of Pmk1 (42 kDa) and Mps1 (46 kDa) MAPK in the WT and indicated PKA-C mutants. Total proteins were extracted from the mycelium of WT and mutants and analyzed with the indicated antibodies. The pmk1 $\Delta$ served as a negative control and the experiment was repeated twice.

of Pmk1 was evident in $\operatorname{cpkA} \Delta$, compared to the increased phosphorylation of Pmk1 in $c p k 2 \Delta$; whereas such activation of Pmk1 MAPK was completely absent in the $c p k A \Delta c p k 2 \Delta$ strain. The level of Mps1 phosphorylation increased in cpkA $\Delta$ or $c p k 2 \Delta$ compared to the WT, but similar to pmk $1 \Delta$ indicating that Mps1 is likely hyperactive in response to/or to compensate for the cell wall defects associated with loss of Pmk1 (Zhao et al., 2005). However, the Mps1 phosphorylation remained stronger in $c p k \mathrm{~A} \Delta$ compared to $c p k 2 \Delta$, likely as a consequence of the weak phosphorylation/activation of Pmk1 in cpkA $\Delta$. The phosphorylation of Mps1 remained unaltered in cpkA $\Delta c p k 2 \Delta$ when compared to WT M. oryzae. These results showed that 

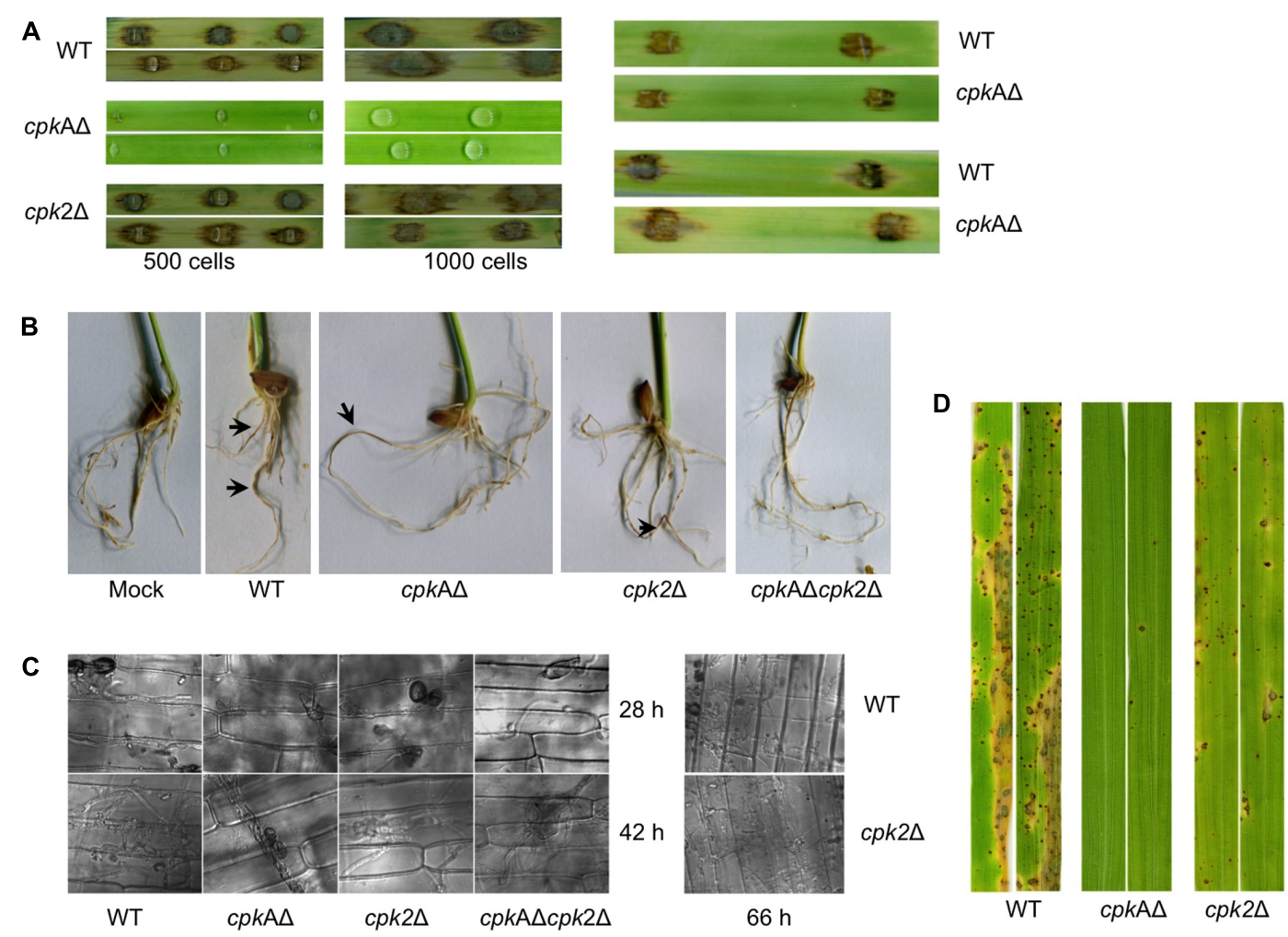

FIGURE 5 | Protein kinase A signaling plays an essential role in the pathogenicity of $M$. oryzae. (A) Barley leaf infection assays with WT and the mutant strains. Conidia from WT or mutant strains were used to inoculate barley leaf explants and the blast disease symptoms/lesions were assessed 7 days post inoculation. Number of conidia used for each inoculation is indicated. For cpkA $\Delta$, the abraded (wounded) leaves showed WT-like blast disease lesions (Right panel). (B) Rice root infection assays with WT and the PKA-C mutant strains. Surface sterilized rice seeds were allowed to germinate and grow on mycelial plugs of the WT or the mutant strains; and necrosis/lesions were recorded after an incubation of 15 days. Mock indicates PA plugs without the fungal cultures. Arrows indicate necrosis/lesions on the roots. (C) Bright field micrographs showing the invasive hyphal growth of WT and PKA-C mutants at the indicated time points when inoculated on rice leaf sheath. Scale bar $=10 \mu \mathrm{m}$. (D) Spray inoculation assays in rice to confirm the pathogenicity of WT and mutants. Conidia (1 $\left.\times 10^{5} / \mathrm{ml}, 5 \mathrm{ml}\right)$ from WT or PKA-C mutants were sprayed on 4-week-old seedlings of rice cultivar CO39. Blast disease symptoms were assessed at $10 \mathrm{dpi}$.

signaling through Pmk1 may be impaired but not completely blocked in cpkA $\Delta$ or $c p k 2 \Delta$, but an additive effect is observed in the double mutant wherein the total loss of Pmk1 activation is likely responsible for the observed defects in appressorium formation.

\section{cAMP-PKA Signaling and Pathogenesis of $M$. oryzae}

We tested the cAMP-PKA mutant strains for their ability to cause blast disease in barley and rice. The $c p k \mathrm{~A} \Delta$ and $c p k \mathrm{~A} \Delta c p k 2 \Delta$ failed to elicit any visible blast symptoms on barley leaves, whereas the WT or $c p k 2 \Delta$ inoculation resulted in typical blast lesions on barley leaves. Wounding of rice or barley leaves with the micropipette tip helped the $c p k \mathrm{~A} \Delta$ to produce WT-like blast lesions in such abraded tissues (Figure 5A). While cpkA $\Delta$ was still able to elicit necrosis on rice roots comparable to the WT or $c p k 2 \Delta$, the $c p k \mathrm{~A} \Delta c p k 2 \Delta$ strain did not produce any visible disease symptoms or necrosis on rice roots (Figure 5B). Rice leaf sheath inoculations revealed that $c p k 2 \Delta$ was pathogenic, and was able to penetrate the host plants ( $28 \mathrm{hpi}$ ), and grow invasively into the neighboring cells similar to the WT (42-72 hpi). Consistent with previous results, the appressoria produced by $c p k \mathrm{~A} \Delta$ were impaired in penetration and could be observed on the surface of the rice leaf sheath at 28 and 42 hpi. The cpkA $\Delta c p k 2 \Delta$ failed to produce appressoria on leaf sheath even at $42 \mathrm{hpi}$ and hence was deemed completely non-pathogenic (Figure 5C). As observed with barley leaf assays, the spray inoculation of conidia from WT or $c p k 2 \Delta$ produced typical blast lesions on rice leaves, while $c p k A \Delta$ remained non-pathogenic (Figure 5D). Since $c p k \mathrm{~A} \Delta c p k 2 \Delta$ produced very few conidia, we were unable to carry out spray inoculation assays for the double mutant strain.

\section{Swapping of the CPKA ORF with CPK2}

In order to check if Cpk2 could functionally complement CpkA, we precisely replaced the CPKA coding sequence with the CPK2 ORF, thus creating a genetic background that consequently lacks $C P K A$, but expresses CPK2 under the CPKA promoter/regulon. The native $C P K 2$ remained unperturbed in such swapped strain. The resultant $C P K A_{\text {Promoter }} C P K 2$ strain showed WT-like vegetative growth, but displayed up to tenfold reduction in conidiation, though the difference between the means was not significant $(p=0.07$; Figures 6A,B). Furthermore, appressorium 


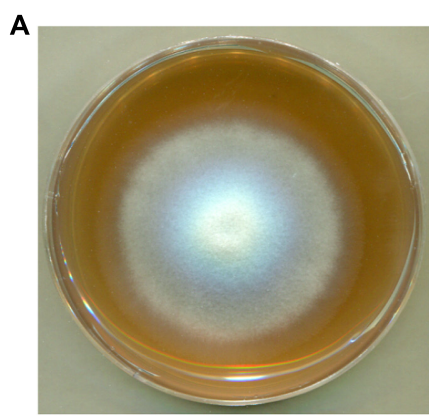

WT

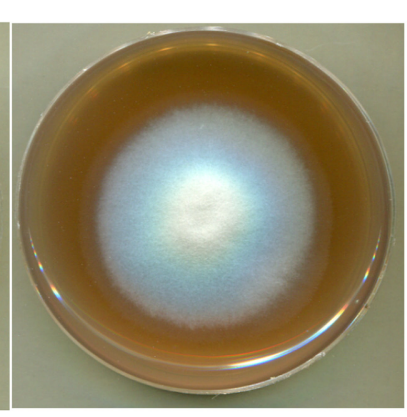

cpkAprocpk2

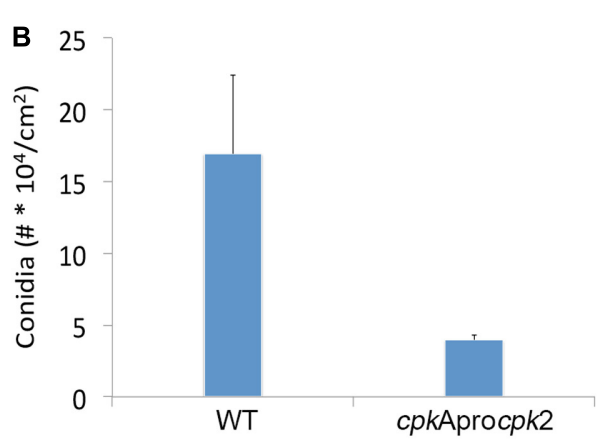

C
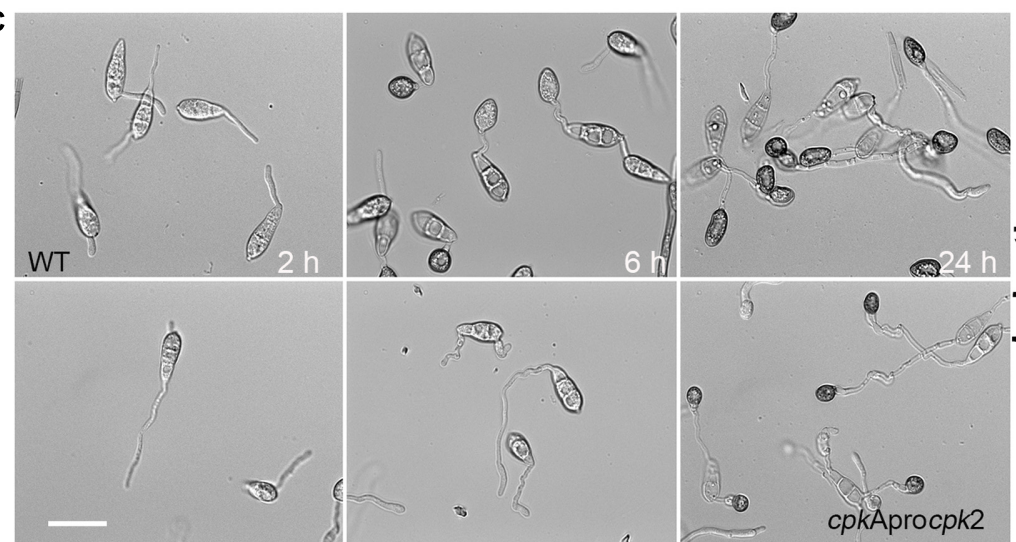

$24 \mathrm{~h}$

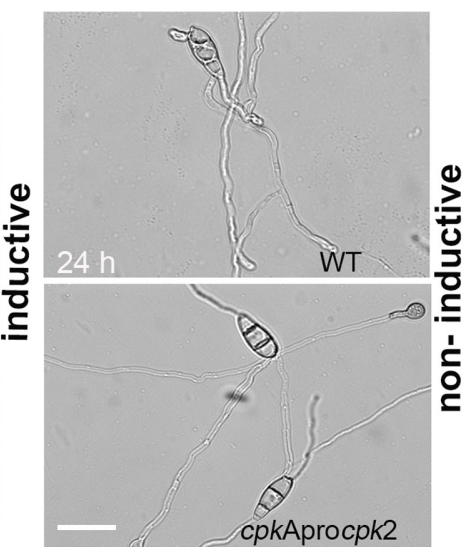

cpkAprocpk2

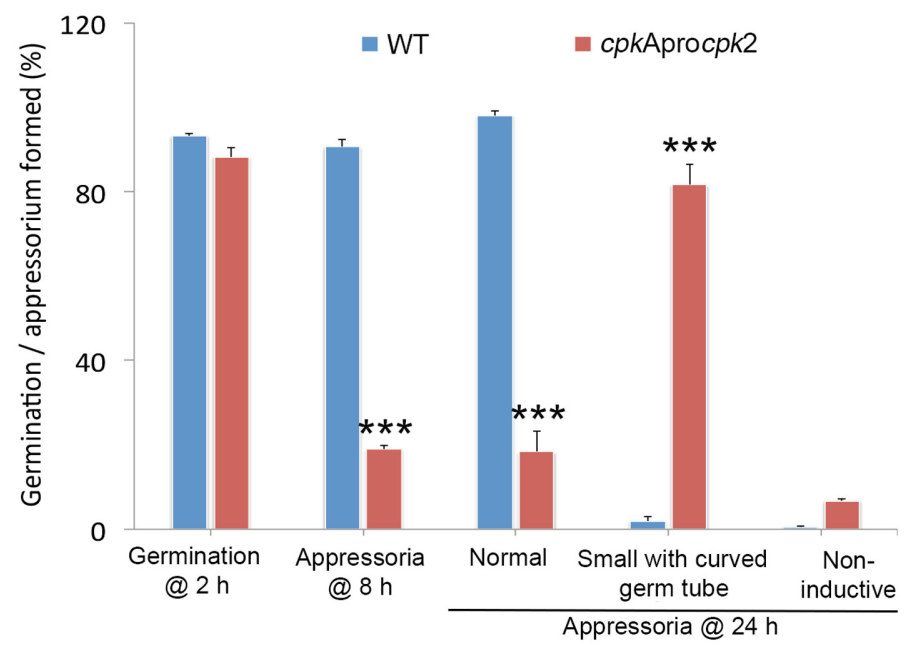

FIGURE 6 | Cpk2 is able to partially compensate for the loss of CpkA. (A) Radial growth of CPKApromoterCPK2 (cpkAprocpk2) compared with WT on prune-agar medium. The CPKApromoterCPK2 strain showed no defects in vegetative growth when compared to the WT M. oryzae. (B) Quantification of conidiation revealed that substituting CPK2 open reading frame for CPKA results in reduced conidiation implicating a major role of CPKA in conidiation. Each bar represents mean \pm SE of three independent replicates. (C) Appressorium formation on inductive (left) and non-inductive surfaces (right) and quantification of germination, appressorium formation by CPKAproCPK2 compared with WT (lower panel). Values represent mean \pm SE of three independent replicates using about 200 conidia per experiment. ${ }^{* * *} p<0.0001$

formation was delayed in the $C P K A_{\text {Promoter }} C P K 2$ strain, and the resultant appressoria formed after prolonged germ tube growth were smaller in size similar to the $c p k A \Delta$ (Figure 6C), again confirming that $C P K A$ is required for proper appressorium formation. The PKA activity could not be detected in the total protein extracts from the mycelia of $C P K A_{\text {Promoter }} C P K 2$ strain.
Interestingly, the $C P K A_{\text {Promoter }} C P K 2$ strain produced typical WT-like blast lesions on rice leaves although the lesion size was smaller than the WT lesions (Figure 7A), thus indicating that the appressorial function is restored to some extent in the $C P K A_{\text {Promoter }} C P K 2$ strain unlike in cpkA $\triangle$. The $C P K A_{\text {Promoter }} C P K 2$ appressoria could penetrate the rice 
A

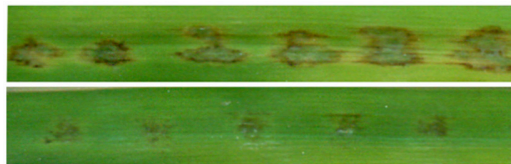

1000 cells

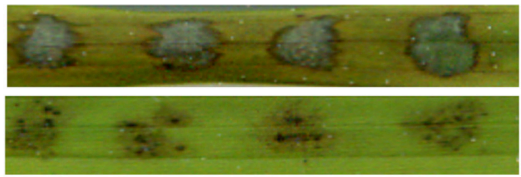

2000 cells
WT

cpkAprocpk2

WT

cpkAprocpk2

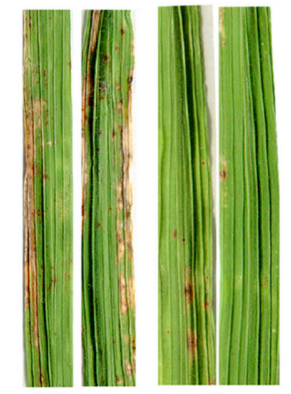

WT cpkAprocpk2

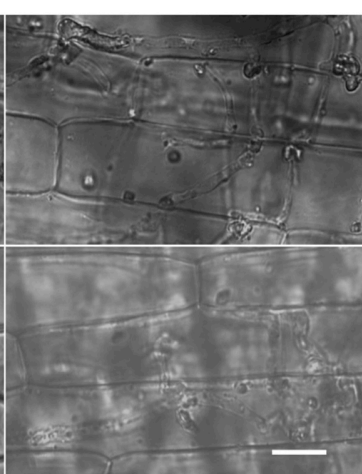

WT

cpkAprocpk2

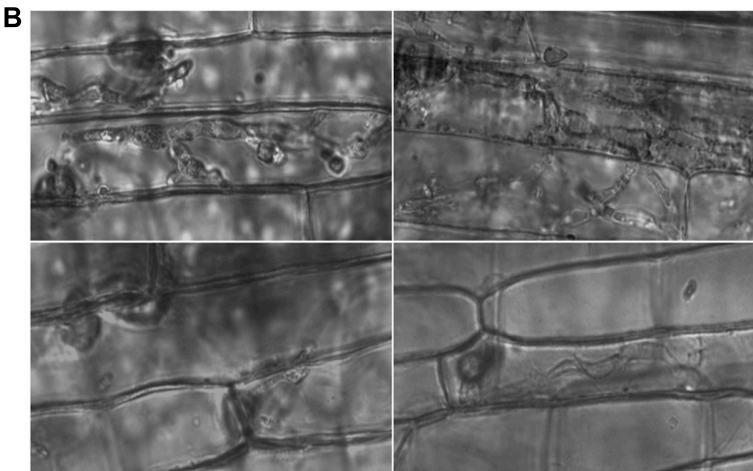

$28 \mathrm{~h}$

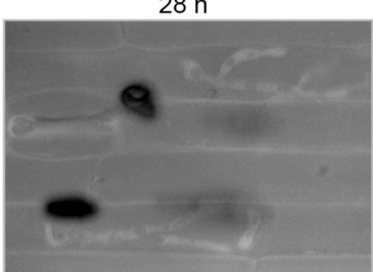

WT
$42 \mathrm{~h}$

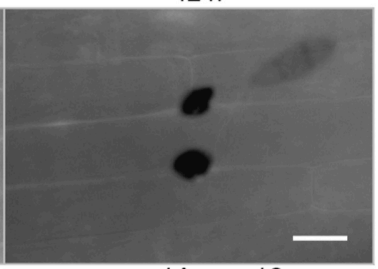

cpkAprocpk2

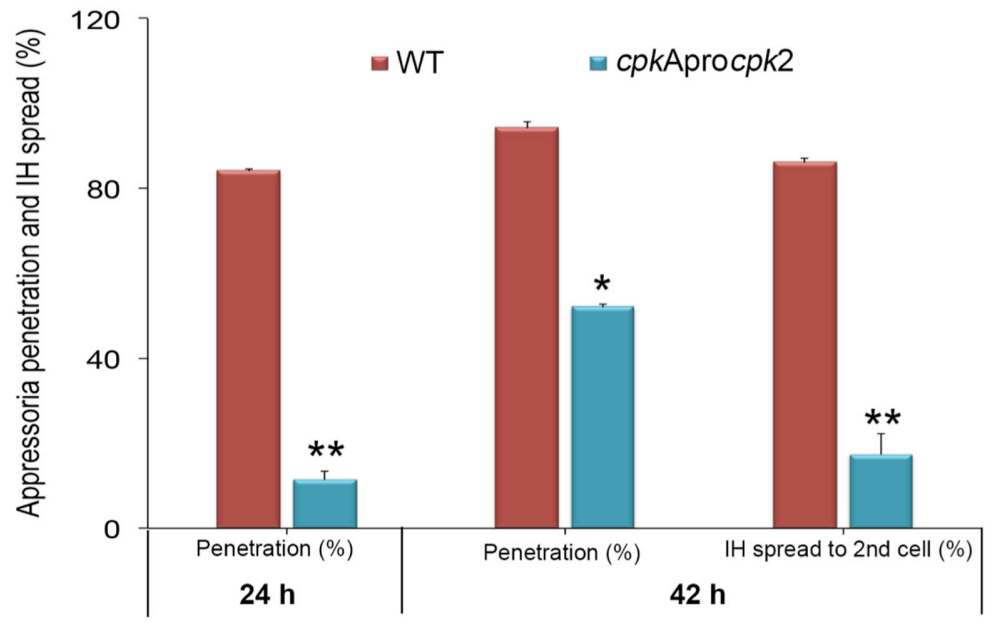

FIGURE 7 | Substituting Cpk2 for CpkA partially suppresses the penetration and pathogenicity defects of cpkA deletion mutant. (A) Infection assays in rice with the conidia of CPKApromoterCPK2 strain compared to the WT. Conidia from WT and CPKApromoterCPK2 were used to spot inoculate rice leaves, or sprayed on to seedlings of rice cultivar $\mathrm{CO} 39$ (right). The number of conidia inoculated on rice leaves is indicated. Disease lesions were scored on $10 \mathrm{dpi}$ for rice leaves and at $14 \mathrm{dpi}$ for the spray inoculation assays. (B) Bright field micrographs showing the invasive hyphal growth of WT and CPKApromoterCPK2 strain at the indicated time points (upper panel). Scale bar $=10 \mu \mathrm{m}$. The CPKApromoterCPK2 showed impaired penetration and was defective in invasive hyphal growth. Micrographs showing host penetration by WT and CPKA Promoter CPK2 at 28h stained with aniline blue to show the callose deposition (lower panel). Bar graph showing the host penetration ability and the percentage of appressoria that formed primary and secondary invasive hyphae from the WT and CPKApromoterCPK2 at the indicated time points (lower right panel). ${ }^{*} p<0.01,{ }^{* *} p<0.001$. 

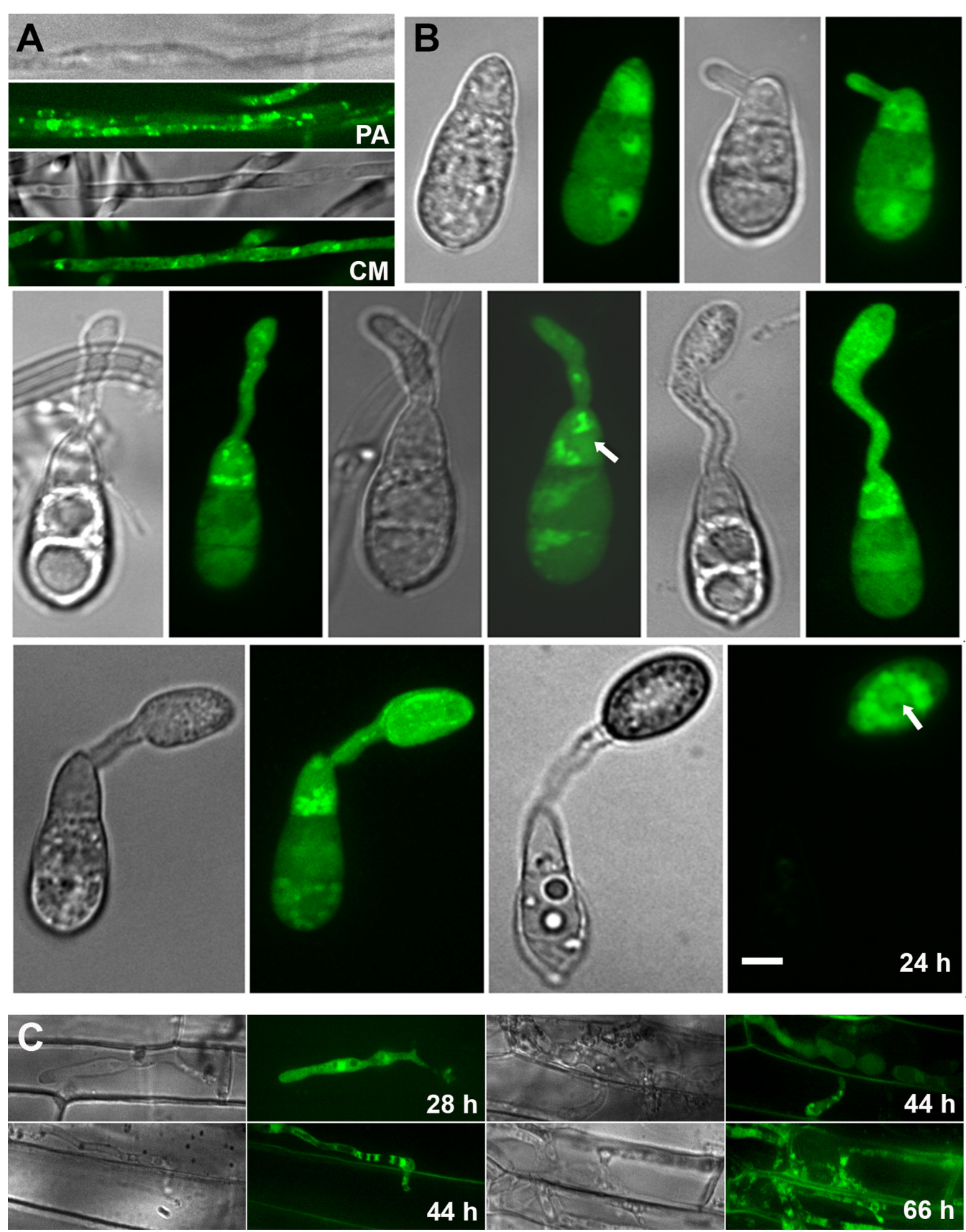

FIGURE 8 | Subcellular localization of the GFP-Cpk2 at different stages of vegetative and asexual development in M. oryzae. (A) Vegetative hyphae and aerial structures from pH3GFP-CPK2 strain were imaged after growth on PA or CM medium for 3 days. (B) Conidia inoculated on the inductive surface were subjected to time lapse analysis and the Bright field and epifluorescent images were captured at the indicated time points using the requisite filters. Images are maximum intensity Z-projections of five confocal stacks, measuring $0.5 \mu \mathrm{m}$ each. Scale bar is $10 \mu \mathrm{m}$. Arrows indicate the nuclear localization of GFP-Cpk2. (C) Subcellular localization of GFP-Cpk2 in the invasive hyphae formed in rice leaf sheath at the indicated time points. Scale bar is $10 \mu \mathrm{m}$.

leaves, albeit delayed compared to the WT, and were able to successfully invade the host plants. However, the IH growth was compromised and the mutant strain remained restricted to the site of inoculation (Figure 7B). Less than $20 \%$ of the $C P K A_{\text {Promoter }} C P K 2$ appressoria could penetrate the rice plants at $28 \mathrm{~h}(p<0.0001)$; and by $42 \mathrm{hpi}$ more than $50 \%$ appressoria could penetrate $(p=0.013)$ and produce IH with only $10 \%$ capable of spread into the neighboring cells. By $42 \mathrm{~h}$, about $80 \%$ of the WT appressoria produced secondary IH ( $p=0.001$; Figure 7 B, bar graph). The cpkA $\Delta$ was able to produce a successful infection when inoculated through wounds, as observed here and in previous reports. In contrast, the $C P K A_{\text {Promoter }} C P K 2$ strain 


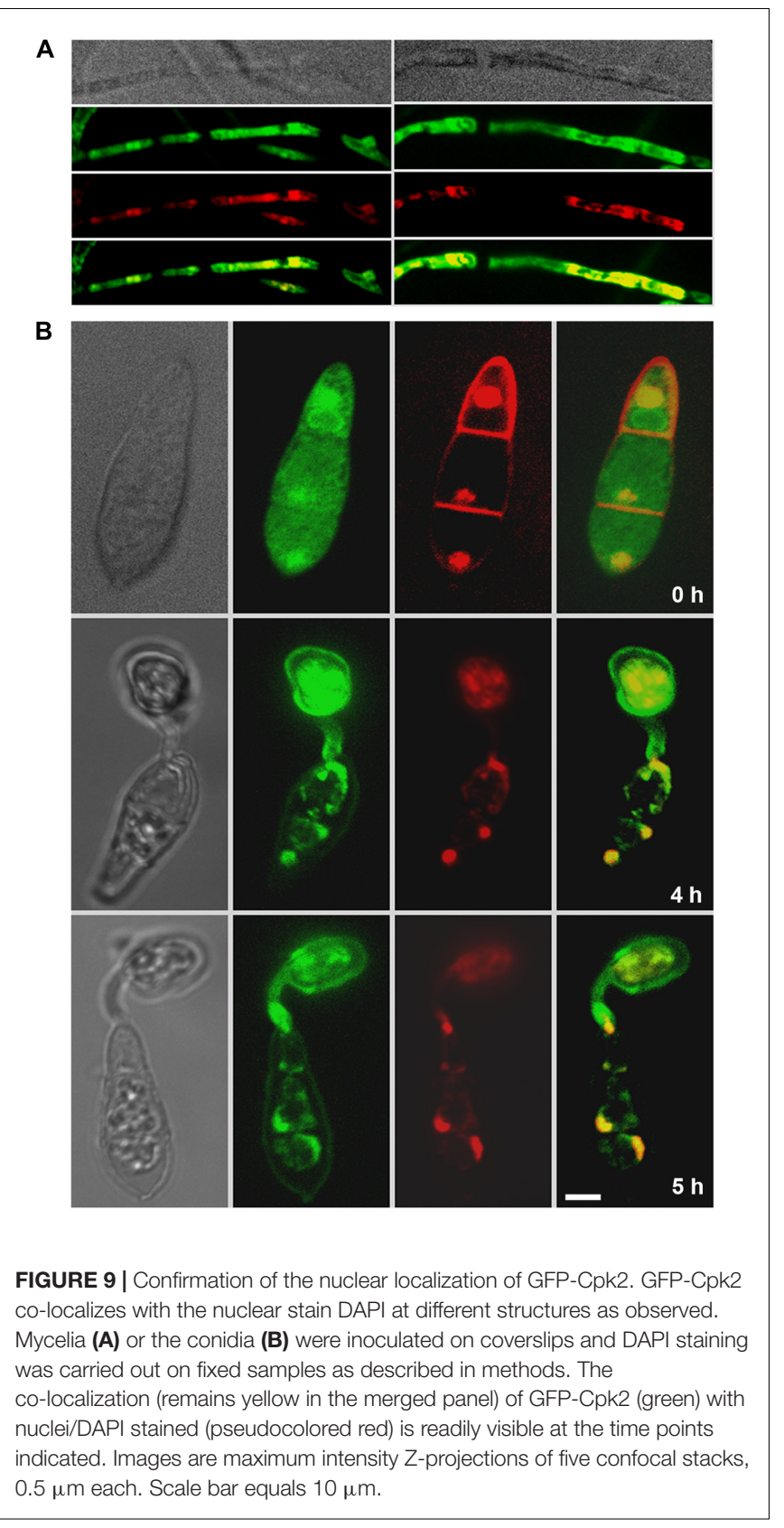

penetrated the rice sheath but was defective in IH growth. To check if the suppression of host penetration results from overexpression of $C P K 2$, we analyzed the transcript levels of $C P K 2$ in the $C P K A_{\text {Promoter }} C P K 2$ strain compared to the WT. The levels of $C P K A$ in the $C P K A_{\text {Promoter }} C P K 2$ strain remained undetectable throughout the vegetative and pathogenic phases thus validating the $c p k \mathrm{~A} \Delta$ strain background. Nevertheless, the results confirmed that $C P K 2$ is not highly expressed in this strain with less than two fold increase at all the phases analyzed, when compared to WT M. oryzae (Supplementary Figure S3). We infer that the suppression of defects associated with loss of $C P K A$ are likely due to the redundancy in Cpk2 function during pathogenic differentiation, and not due to an increase in CPK2 transcript levels per se in the swapped strain. To conclude, Cpk2 shares a potentially redundant function with $\mathrm{CpkA}$, but is unable to fully compensate/complement the loss of CPKA activity in M. oryzae.

\section{Cpk2-GFP Localizes to Dynamic Cytoplasmic Vesicles, and the Nucleus}

To analyze the subcellular localization of Cpk2 during asexual and pathogenic phases of $M$. oryzae, the Cpk2 was fused with GFP at its C terminus. The expression from the native Cpk2 promoter was too weak to observe proper epifluorescence (Supplementary Figure S4). Hence, the GFP-Cpk2 fusion was expressed under the control of the constitutive Histone $\mathrm{H} 3$ promoter ( $p H 3 G F P-C P K 2)$. The in vivo functionality of the fusion protein was verified through rigorous analysis of several phenotypes, and the aforementioned modified strains were found to be comparable to the parental untagged strain in all aspects of growth and pathogenicity (Supplementary Figure S5). A double-tagged strain, CPK2-GFP CPKA$m$ Cherry, was generated to examine the colocalization (if any).

The Cpk2-GFP was highly expressed in the vegetative stage, i.e., in mycelia/aerial hyphae on PA medium, compared to conidia and related structures therein (Supplementary Figures S4B,C). Staining with the Hoechst dye confirmed the nuclear localization of Cpk2-GFP during vegetative growth and also in the conidiophores (Supplementary Figure S4D). However, such significant colocalization was not evident in the developing conidia due to weak Cpk2GFP signal therein. The constitutively expressed GFP-Cpk2 (H3ProGFP-CPK2 strain) showed a similar localization pattern, i.e., remained nuclear and cytoplasmic during mycelial growth. Nuclear localization of GFP-Cpk2 was clearly evident in conidia, and as cytoplasmic vesicles in the terminal cell of the conidia and also in germ tubes (Figure 8). The GFP-Cpk2 vesicles moved to the emerging appressorium at the hooking stage. In mature appressoria (24 h), GFP-Cpk2 showed a peri-nuclear vesicular localization, although the nuclear localization per se was not as prominent as in conidia (Figure 8). In order to confirm the nuclear localization of GFP-Cpk2, the conidia from H3ProGFP-CPK2 strain were co-stained with DAPI. GFP-Cpk2 colocalized with the DAPI signal, thus confirming the nuclear localization of Cpk2 therein (Figure 9). The Cpk2-GFP vesicles co-localized with the CpkA-mCherry vesicles in the cytoplasm at different stages analyzed. However, nuclear localization of Cpk2-GFP was not clear in this strain likely because of the weak Cpk2GFP signal due to native expression, and/or due to masking by the stronger CpkA-mC expression (Figure 10). We conclude that $\mathrm{Cpk} 2$ is compartmentalized in the nucleus, and its colocalization with CpkA is exclusive to the cytoplasmic vesicles. We infer that such intracellular localization facilitates RpkA interaction with both $\mathrm{Cpk} 2$ and $\mathrm{CpkA}$, thus enabling robust cAMP-PKA enzyme activity/function to be regulated effectively in a compartmentalized manner. Lastly, the localization pattern clearly supports some special targets (and/or functions) for Cpk2 in activating the downstream cyclic AMP signaling in 

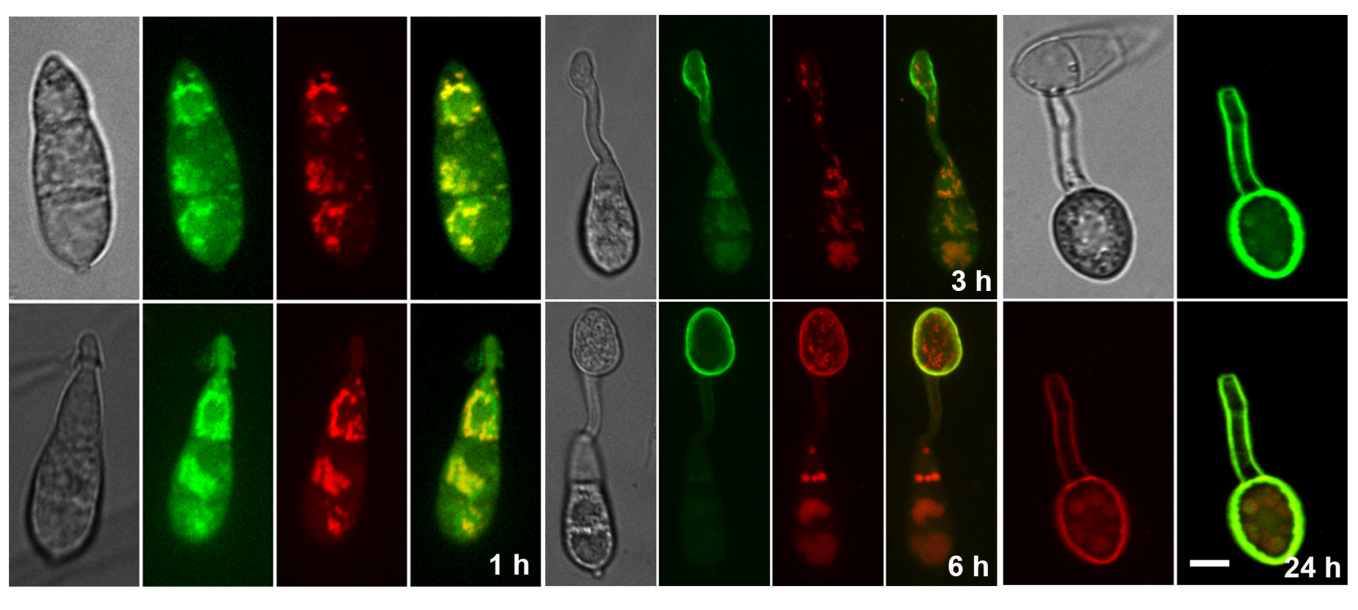

FIGURE 10 | Co-localization of Cpk2-GFP with CPKA-mcherry. Cpk2-GFP cytoplasmic vesicles colocalize with CpkA-mCherry in the conidia and germ tubes. In the appressoria (24 h) a weak co-localization is evident in the vesicular structures within the perinuclear region.

the nucleus during pathogenic differentiation in the rice blast fungus.

\section{DISCUSSION}

Most filamentous fungi examined to date, contain multiple isoforms of the catalytic cAMP-PKA subunit, with one such isoform playing a predominant role in growth and development. Despite its importance in regulating biological features and pathogenicity, the PKA catalytic subunits are not essential for cell viability in a number of fungal species (Dürrenberger et al., 1998; D'Souza and Heitman, 2001; Fuller et al., 2011). However, at least one TPK gene is required for cell viability and the tpk1 tpk2 tpk3 triple mutant is not viable in S. cerevisiae (Toda et al., 1987). In A. nidulans, deletion of both $p k a B$ and $p k a A$ is lethal, though overexpression of $p k a B$ can suppress some defects caused by $\triangle p k a A$, indicating the overlapping roles of PkaA and PkaB (Ni et al., 2005). U. maydis and F. graminearum represent only a minority of plant pathogens wherein the effect of the loss of both the PKA-C isoforms has been analyzed (Dürrenberger et al., 1998; Hu et al., 2014). M. oryzae contains a divergent PKA-C isoform, Cpk2, but its contribution to growth and pathogenesis remained unexplored largely due to the earlier indication that the cpkAcpk2 double mutant is likely inviable (Choi and $\mathrm{Xu}$, 2010). A major challenge in deciphering the overall cAMP-PKA signaling is to fully understand the contribution(s) of both the catalytic subunits, CpkA and Cpk2, in M. oryzae. Previous studies revealed the importance of CPKA in appressorium formation and pathogenicity in M. oryzae (Mitchell and Dean, 1995; Xu et al., 1997; Selvaraj et al., 2017). Our study provides a detailed elucidation of the function, dynamics and organization of the individual signaling components, CpkA and Cpk2 of the cAMP-PKA signaling in M. oryzae. Here, we showed that the cAMP-PKA signaling is not required for viability per se in M. oryzae, but is necessary for proper growth, conidiation and pathogenicity. This further adds to the previous findings that
MAC1 or RPKA are dispensable for viability but essential for pathogenic differentiation (Choi and Dean, 1997; Selvaraj et al., 2017). Our study also complements a recent elegant analysis (Li Y. et al., 2017) of an important downstream transcriptional target/effector of PKA-C, MoSFL1, which was identified as a spontaneous suppressor in the double deletion mutant of the cAMP-PKA catalytic subunits. In addition, we have provided a detailed functional analysis and subcellular localization of $\mathrm{Cpk} 2$, and gained insight into the functional interdependency between the two PKA-C subunits, which had remained unelucidated thus far in M. oryzae.

In $M$. oryzae, CpkA plays a role in appressorium morphogenesis and plant infection though it is dispensable for vegetative growth and conidiation, and is predicted to contribute the major PKA catalytic function (Mitchell and Dean, 1995; Xu et al., 1997). Consistent with this observation, deletion of CPK2 had no detectable effect on conidiation, appressorium formation or pathogenicity, thus making it redundant in function with CPKA. The enzyme activities and relative expression of the two isoforms in WT and mutant backgrounds revealed that cells do not compensate for the loss of one C isoform by overexpression of the other as in S. cerevisiae (Mazón et al., 1993; Robertson et al., 2000) and their interactions are certainly not regulated at the transcriptional level. However, we have shown here the functional capacity of Cpk2 to act in concert with CpkA in the regulation of vegetative growth and conidiation in M. oryzae. Appressorium induction upon proper surface sensing is a crucial step for pathogenesis in M. oryzae, and our results reflect the importance of $\mathrm{Cpk} 2$ function in this process. Our findings suggest that $\mathrm{Cpk} 2$, in addition to acting as an inducer of appressorium formation in concert with CpkA, also plays a regulatory role in suppressing appressoria biogenesis under unfavorable conditions such as on hydrophilic surfaces.

The defects of PKA-C null mutant $(c p k A \Delta c p k 2 \Delta)$ resembled the phenotype of mac1 $\Delta$ (Choi and Dean, 1997), despite the significantly higher accumulation of cAMP in this strain 
and also in individual mutants of the PKA-C subunits. In S. cerevisiae and Cryptococcus neoformans, it has been shown that the PKA-C regulate cAMP levels through a negative feedback loop by activating PDEs (Ma et al., 1999). Preliminary results on cAMP levels in PKA mutants showed that such feedback inhibition on intracellular cAMP levels via $\mathrm{PdeH}$ occurs in $M$. oryzae too. Further, the downregulation of Pmk1 phosphorylation in the PKA-C mutants implies that the crosstalk between the cAMP and MAPK signaling might occur at the level of PKA-C. Although not characterized fully in this study, the defects in $c p k A \Delta c p k 2 \Delta$ related to surface sensing and response, appear similar to the suppressor mutant phenotypes in the CHM1-deletion mutant (Li et al., 2004). The molecular identity of such suppressor(s) of $\operatorname{chm} 1 \Delta$ has not been ascertained yet. The $\operatorname{chm} 1 \Delta$ also showed additional defects in hyphal growth, conidiation and appressorium formation, which could not be suppressed by exogenous cAMP (Li et al., 2004).

Interestingly, we did not detect phosphorylation of the synthetic PKA substrate, kemptide, in any of the strains in which CPKA was deleted. Thus, Cpk2 is unable to serve as the primary PKA-C in $M$. oryzae. The Cpk2 overlap provides a basal level of PKA-C function to allow efficient vegetative growth and to maintain turgor for penetration of appressoria, but not inducible PKA function sufficient for conidiation and appressorium morphogenesis or proper IH growth. Nevertheless, the localization of Cpk2-GFP clearly implies some special functions for CPK2 in $M$. oryzae. It is well recognized that compartmentalization of cAMP signaling allows spatially distinct pools of PKA to be differentially activated. PKA isoforms are anchored at specific intracellular sites by A-kinase anchoring proteins (AKAPs) in mammalian cells (Smith and Scott, 2006). However, AKAPs are not present in fungi. We showed earlier that cAMP signaling is compartmentalized in the nucleus and cytoplasm in M. oryzae (Ramanujam and Naqvi, 2010). The RpkA localizes to the nucleus whereas CpkA is present predominantly on cytoplasmic vesicles with the PKA holoenzyme being cytosolic (Selvaraj et al., 2017). We infer that the nuclear pool of Cpk2-GFP is a consequence of its association with RpkA therein, and that this interaction drives the nuclear function of cAMP signaling in M. oryzae. The primary locale for CpkA being vesicular structures in the cytoplasm; and the RpkA and Cpk2 being in the cytoplasm and nucleus allows discrete cAMP-PKA modules that respond to distinct intracellular cAMP pools and subsequently modify specific target proteins. Furthermore, the compartmentalization of cAMP PDEs, the PdeH and PdeL to the cytoplasm and nucleus respectively (Ramanujam and Naqvi, 2010) also shows the importance of tailoring individual cAMP responses to precisely modulate the downstream signaling cascade.

\section{CONCLUSION}

Proper PKA-C signaling is essential for the invasive growth and pathogenicity, and the balanced activities of the CpkA and Cpk2 isoforms likely plays important roles in robust regulation of the infection process in $M$. oryzae. CpkA being the primary PKA, Cpk2 maintains important function(s) in regulating vegetative growth, conidiation and appressorium formation and also contributes to the spatial and temporal regulation of cAMP-PKA signaling in $M$. oryzae. CpkA and Cpk2 act in a redundant as well as parallel/specific manner to activate the downstream effectors of the cyclic AMP signaling and also Pmk1 MAPK during initiation and spread of the blast disease in rice. Future studies will focus on analyzing the differential regulation and downstream targets of the two PKA-C isoforms in the rice blast pathosystem.

\section{AUTHOR CONTRIBUTIONS}

Conceived and designed the experiments: NN. Performed the experiments: PS, QS, and FY. Analyzed the data: PS and NN. Contributed reagents/materials/analysis tools: NN. Wrote the paper: PS and NN.

\section{FUNDING}

Research in the Naqvi group is funded by the Temasek Life Sciences Laboratory (Singapore) and The National Research Foundation (Prime Minister's Office, Grant No. NRF-CRP7-2010-02 and NRF-CRP16-2015-04), Singapore.

\section{ACKNOWLEDGMENTS}

We are grateful to the Fungal Patho-biology Group at TLL for discussions and suggestions. We thank M. Madhaiyan for help in organizing the figure panels using Photoshop. We are grateful to $\mathrm{Xu}$ Jin-Rong for sharing unpublished data on CPK2.

\section{SUPPLEMENTARY MATERIAL}

The Supplementary Material for this article can be found online at: https://www.frontiersin.org/articles/10.3389/fmicb.2017. 02289/full\#supplementary-material

FIGURE S1 | Amino acid sequence alignment for the PKA-C subunits, CpkA and Cpk2, from M. oryzae. The protein sequences of CpkA and Cpk2 showing identities (shaded black) and similarities (gray background). Alignment was carried out using ClustalW of the MegAlign software, a program of the Lasergene package (DNASTAR) using default parameters; and shaded with BOXSHADE 3.2.1.

FIGURE S2 | Construction of deletion mutants in CPKA and CPK2 mutants and confirmation of the transformants. (A) Schematic representation of CPKA (left) and CPK2 (right) loci, and the deletion mutant strategy in $M$. oryzae. The open reading frame of the genes was deleted and replaced with the Glufosinate ammonium (BAR) or hygromycin resistance (HPH) cassette. Solid bars and short open boxes represent coding regions and introns, respectively; whereas dashed lines indicate the genomic flanks used for gene targeting. Restriction endonuclease cleavage sites (E- EcoRI, B- BamHI, P-Pstl, H-HindIII). (B) Southern blot analysis of the genomic DNA of WT and transformants for CPKA (1-5) (left) and CPK2 (1-7) (right) transformants. (C) Locus specific PCR confirming the proper integration of cpk2 deletion in cpkA $\Delta$ background. 
FIGURE S3 | The CPK2 is not overexpressed in WT when driven with the CPKA promoter. Bar graph showing the fold change in the transcript levels of CPKA and CPK2 in the CPKApromoterCPK2 strain at different time points analyzed during vegetative or pathogenic growth. Fold change in gene expression was calculated from the average of three independent measurements, with $\beta$-Tubulin of $M$. oryzae as internal control and normalized to WT CPKA and CPK2 levels at the respective time points.

FIGURE S4 I The Cpk2-GFP is weakly expressed with the native promoter. (A) Schematic representation showing the in-frame integration of GFP within the CPK2 genomic locus; and the locus-specific PCR of the transformants confirming

\section{REFERENCES}

Adachi, K., and Hamer, J. E. (1998). Divergent cAMP signaling pathways regulate growth and pathogenesis in the rice blast fungus Magnaporthe grisea. Plant Cell 10, 1361-1374. doi: 10.1105/tpc.10.8.1361

Altschul, S. F., Madden, T. L., Schaffer, A. A., Zhang, J., Zhang, Z., Miller, W., et al. (1997). Gapped BLAST and PSI-BLAST: a new generation of protein database search programs. Nucleic Acids Res. 25, 3389-3402. doi: 10.1093/nar/25.17.3389

Banno, S., Ochiai, N., Noguchi, R., Kimura, M., Yamaguchi, I., Kanzaki, S.-I., et al. (2005). A catalytic subunit of cyclic AMP-dependent protein kinase, PKAC-1, regulates asexual differentiation in Neurospora crassa. Genes Genet. Syst. 80, 25-34. doi: 10.1266/ggs.80.25

Bockmühl, D. P., Krishnamurthy, S., Gerads, M., Sonneborn, A., and Ernst, J. F. (2001). Distinct and redundant roles of the two protein kinase A isoforms Tpk1p and Tpk2p in morphogenesis and growth of Candida albicans. Mol. Microbiol. 42, 1243-1257. doi: 10.1046/j.1365-2958.2001.02688.x

Bosch, D. E., Willard, F. S., Ramanujam, R., Kimple, A. J., Willard, M. D., Naqvi, N. I., et al. (2012). A P-loop mutation in G $\alpha$ subunits prevents transition to the active state: implications for G-protein signaling in fungal pathogenesis. PLOS Pathog. 8:e1002553. doi: 10.1371/journal.ppat.1002553

Bruno, K. S., Tenjo, F., Li, L., Hamer, J. E., and Xu, J. R. (2004). Cellular localization and role of kinase activity of PMK1 in Magnaporthe grisea. Eukaryot. Cell 3, 1525-1532. doi: 10.1128/EC.3.6.1525-1532.2004

Choi, W., and Dean, R. A. (1997). The adenylate cyclase gene MAC1 of Magnaporthe grisea controls appressorium formation and other aspects of growth and development. Plant Cell 9, 1973-1983. doi: 10.1105/tpc.9.11.1973

Choi, Y.-E., and Xu, J.-R. (2010). The cAMP signaling pathway in Fusarium verticillioides is important for conidiation, plant infection, and stress responses but not fumonisin production. Mol. Plant Microbe Interact. 23, 522-533. doi: 10.1094/MPMI-23-4-0522

Cloutier, M., Castilla, R., Bolduc, N., Zelada, A., Martineau, P., Bouillon, M., et al. (2003). The two isoforms of the cAMP-dependent protein kinase catalytic subunit are involved in the control of dimorphism in the human fungal pathogen Candida albicans. Fungal Genet. Biol. 38, 133-141. doi: 10.1016/ S1087-1845(02)00520-0

Dean, R. A., Talbot, N. J., Ebbole, D. J., Farman, M. L., Mitchell, T. K., Orbach, M. J., et al. (2005). The genome sequence of the rice blast fungus Magnaporthe grisea. Nature 434, 980-986. doi: 10.1038/nature03449

Deng, Y. Z., Qu, Z., He, Y., and Naqvi, N. I. (2012). Sorting nexin Snx41 is essential for conidiation and mediates glutathione-based antioxidant defense during invasive growth in Magnaporthe oryzae. Autophagy 8, 1058-1070. doi: 10.4161 /auto. 20217

D’Souza, C. A., and Heitman, J. (2001). Conserved cAMP signaling cascades regulate fungal development and virulence. FEMS Microbiol. Rev. 25, 349-364. doi: 10.1111/j.1574-6976.2001.tb00582.x

Dufresne, M., and Osbourn, A. E. (2001). Definition of tissue-specific and general requirements for plant infection in a phytopathogenic fungus. Mol. Plant Microbe Interact. 14, 300-307. doi: 10.1094/MPMI.2001.14.3.300

Dürrenberger, F., Wong, K., and Kronstad, J. W. (1998). Identification of a cAMP-dependent protein kinase catalytic subunit required for virulence and morphogenesis in Ustilago maydis. Proc. Natl. Acad. Sci. U.S.A. 95, 5684-5689. doi: $10.1073 /$ pnas.95.10.5684

Fang, E. G., and Dean, R. A. (2000). Site-directed mutagenesis of the magB gene affects growth and development in Magnaporthe grisea. Mol. Plant Microbe Interact. 13, 1214-1227. doi: 10.1094/MPMI.2000.13.11.1214 the requisite integration. (B) Confocal images showing the cytoplasmic localization of Cpk2-GFP in the hyphae grown in Prune agar or Complete medium. (C) Cytoplasmic and nuclear localization of Cpk2-GFP in conidia at different stages of pathogenic differentiation. Arrows indicate nuclear localization of the Cpk2-GFP. (D) Images showing colocalization of Cpk2-GFP (green) with the Hoechst stain (nuclear marker - pseudocolored in blue) in the mycelia and conidiophore.

FIGURE S5 | Characterization of GFP-CPK2 strain. The strain H3ProGFP-CPK2 forms appressoria on inductive and non-inductive surfaces (A) and able to penetrate the rice leaf sheath and produce the infection similar to the WT M. oryzae (B).

Fuller, K. K., Richie, D. L., Feng, X., Krishnan, K., Stephens, T. J., WikenheiserBrokamp, K. A., et al. (2011). Divergent Protein Kinase A isoforms co-ordinately regulate conidial germination, carbohydrate metabolism and virulence in Aspergillus fumigatus. Mol. Microbiol. 79, 1045-1062. doi: 10.1111/ j.1365-2958.2010.07509.x

Hanks, S. K., and Hunter, T. (1995). Protein kinases 6. The eukaryotic protein kinase superfamily: kinase (catalytic) domain structure and classification. FASEB J. 9, 576-596.

Hao, Z., Tong, Y., Han, Y., Wu, D., Yang, Z., Shen, S., et al. (2015). Molecular characterization of StpkaC2 and expression patterns of both PKA-c isoforms during the invasive growth of Setosphaeria turcica. Trop. Plant Pathol. 40, 244-250. doi: 10.1007/s40858-015-0041-9

Hu, S., Zhou, X., Gu, X., Cao, S., Wang, C., and Xu, J.-R. (2014). The cAMP-PKA pathway regulates growth, sexual and asexual differentiation, and pathogenesis in Fusarium graminearum. Mol. Plant Microbe Interact. 27, 557-566. doi: 10.1094/MPMI-10-13-0306-R

Kang, S. H., Khang, C. H., and Lee, Y.-H. (1999). Regulation of cAMPdependent protein kinase during appressorium formation in Magnaporthe grisea. FEMS Microbiol. Lett. 170, 419-423. doi: 10.1111/j.1574-6968.1999. tb13403.x

Kankanala, P., Czymmek, K., and Valent, B. (2007). Roles for rice membrane dynamics and plasmodesmata during biotrophic invasion by the blast fungus. Plant Cell 19, 706-724. doi: 10.1105/tpc.106.046300

Kou, Y., Tan, Y. H., Ramanujam, R., and Naqvi, N. I. (2016). Structure-function analyses of the Pth11 receptor reveal an important role for CFEM motif and redox regulation in rice blast. New Phytol. 214, 330-342. doi: 10.1111/nph. 14347

Lee, N., D'Souza, C. A., and Kronstad, J. W. (2003). Of smuts, blasts, mildews, and blights: cAMP signaling in phytopathogenic fungi. Annu. Rev. Phytopathol. 41, 399-427. doi: 10.1146/annurev.phyto.41.052002.095728

Lengeler, K. B., Davidson, R. C., D’souza, C., Harashima, T., Shen, W.-C., Wang, P., et al. (2000). Signal transduction cascades regulating fungal development and virulence. Microbiol. Mol. Biol. Rev. 64, 746-785. doi: 10.1128/MMBR.64.4.746785.2000

Li, C., Cao, S., Zhang, C., Zhang, Y., Zhang, Q., Xu, J. R., et al. (2017). MoCDC14 is important for septation during conidiation and appressorium formation in Magnaporthe oryzae. Mol. Plant Pathol. doi: 10.1111/mpp.12523 [Epub ahead of print].

Li, G., Zhou, X., and Xu, J.-R. (2012). Genetic control of infection-related development in Magnaporthe oryzae. Curr. Opin. Microbiol. 15, 678-684. doi: 10.1016/j.mib.2012.09.004

Li, L., Xue, C., Bruno, K., Nishimura, M., and Xu, J.-R. (2004). Two PAK kinase genes, CHM1 and MST20, have distinct functions in Magnaporthe grisea. Mol. Plant Microbe Interact. 17, 547-556. doi: 10.1094/MPMI.2004.17. 5.547

Li, Y., Zhang, X., Hu, S., Liu, H., and Xu, J.-R. (2017). PKA activity is essential for relieving the suppression of hyphal growth and appressorium formation by MoSfl1 in Magnaporthe oryzae. PLOS Genet. 13:e1006954. doi: 10.1371/journal. pgen.1006954

Liebmann, B., Müller, M., Braun, A., and Brakhage, A. A. (2004). The cyclic AMP-dependent protein kinase a network regulates development and virulence in Aspergillus fumigatus. Infect. Immun. 72, 5193-5203. doi: 10.1128/IAI.72.9. 5193-5203.2004

Liu, H., Suresh, A., Willard, F. S., Siderovski, D. P., Lu, S., and Naqvi, N. I. (2007). Rgs1 regulates multiple Galpha subunits in Magnaporthe pathogenesis, 
asexual growth and thigmotropism. EMBO J. 26, 690-700. doi: 10.1038/sj. emboj.7601536

Liu, S., and Dean, R. A. (1997). G protein $\alpha$ subunit genes control growth, development, and pathogenicity of Magnaporthe grisea. Mol. Plant Microbe Interact. 10, 1075-1086. doi: 10.1094/MPMI.1997.10. 9.1075

Liu, W., Liu, J., Ning, Y., Ding, B., Wang, X., Wang, Z., et al. (2013). Recent progress in understanding PAMP-and effector-triggered immunity against the rice blast fungus Magnaporthe oryzae. Mol. Plant 6, 605-620. doi: 10.1093/mp/ sst015

Liu, W., Zhou, X., Li, G., Li, L., Kong, L., Wang, C., et al. (2011). Multiple plant surface signals are sensed by different mechanisms in the rice blast fungus for appressorium formation. PLOS Pathog. 7:e1001261. doi: 10.1371/journal.ppat. 1001261

Ma, P., Wera, S., Van Dijck, P., and Thevelein, J. M. (1999). The PDE1-encoded low-affinity phosphodiesterase in the yeast Saccharomyces cerevisiae has a specific function in controlling agonist-induced cAMP signaling. Mol. Biol. Cell 10, 91-104. doi: 10.1091/mbc.10.1.91

Mazón, M. J., Behrens, M. M., Morgado, E., and Portillo, F. (1993). Low activity of the yeast cAMP-dependent protein kinase catalytic subunit Tpk3 is due to the poor expression of the TPK3 gene. FEBS J. 213, 501-506. doi: 10.1111/j.14321033.1993.tb17787.x

Mehrabi, R., and Kema, G. H. (2006). Protein kinase A subunits of the ascomycete pathogen Mycosphaerella graminicola regulate asexual fructification, filamentation, melanization and osmosensing. Mol. Plant Pathol. 7, 565-577. doi: 10.1111/j.1364-3703.2006.00361.x

Mitchell, T. K., and Dean, R. A. (1995). The cAMP-dependent protein kinase catalytic subunit is required for appressorium formation and pathogenesis by the rice blast pathogen Magnaporthe grisea. Plant Cell 7, 1869-1878. doi: $10.1105 /$ tpc.7.11.1869

Nalley, L., Tsiboe, F., Durand-Morat, A., Shew, A., and Thoma, G. (2016). Economic and environmental impact of rice blast pathogen (Magnaporthe oryzae) alleviation in the United States. PLOS ONE 11:e0167295. doi: 10.1371/ journal.pone.0167295

Ni, M., Rierson, S., Seo, J.-A., and Yu, J.-H. (2005). The pkaB gene encoding the secondary protein kinase A catalytic subunit has a synthetic lethal interaction with pkaA and plays overlapping and opposite roles in Aspergillus nidulans. Eukaryot. Cell 4, 1465-1476. doi: 10.1128/EC.4.8.1465-1476. 2005

Nishimura, M., Park, G., and Xu, J. R. (2003). The G-beta subunit MGB1 is involved in regulating multiple steps of infection-related morphogenesis in Magnaporthe grisea. Mol. Microbiol. 50, 231-243. doi: 10.1046/j.1365-2958.2003. 03676.x

Pan, X., and Heitman, J. (1999). Cyclic AMP-dependent protein kinase regulates pseudohyphal differentiation in Saccharomyces cerevisiae. Mol. Cell. Biol. 19, 4874-4887. doi: 10.1128/MCB.19.7.4874

Patkar, R. N., Ramos-Pamplona, M., Gupta, A. P., Fan, Y., and Naqvi, N. I. (2012). Mitochondrial $\beta$-oxidation regulates organellar integrity and is necessary for conidial germination and invasive growth in Magnaporthe oryzae. Mol. Microbiol. 86, 1345-1363. doi: 10.1111/mmi.12060

Patkar, R. N., Suresh, A., and Naqvi, N. I. (2010). MoTea4-mediated polarized growth is essential for proper asexual development and pathogenesis in Magnaporthe oryzae. Eukaryot. Cell 9, 1029-1038. doi: 10.1128/EC. 00292-09

Pearce, L. R., Komander, D., and Alessi, D. R. (2010). The nuts and bolts of AGC protein kinases. Nat. Rev. Mol. Cell Biol. 11, 9-22. doi: 10.1038/ nrm 2822

Ramanujam, R., Calvert, M. E., Selvaraj, P., and Naqvi, N. I. (2013). The late endosomal HOPS complex anchors active G-protein signaling essential for pathogenesis in Magnaporthe oryzae. PLOS Pathog. 9:e1003527. doi: 10.1371/ journal.ppat.1003527

Ramanujam, R., and Naqvi, N. I. (2010). PdeH, a high-affinity cAMP phosphodiesterase, is a key regulator of asexual and pathogenic differentiation in Magnaporthe oryzae. PLOS Pathog. 6:e1000897. doi: 10.1371/journal.ppat. 1000897

Ramanujam, R., Yishi, X., Liu, H., and Naqvi, N. I. (2012). Structurefunction analysis of Rgs1 in Magnaporthe oryzae: role of DEP domains in subcellular targeting. PLOS ONE 7:e41084. doi: 10.1371/journal.pone.004 1084

Robertson, L. S., Causton, H. C., Young, R. A., and Fink, G. R. (2000). The yeast A kinases differentially regulate iron uptake and respiratory function. Proc. Natl. Acad. Sci. U.S.A. 97, 5984-5988. doi: 10.1073/pnas.100113397

Robertson, L. S., and Fink, G. R. (1998). The three yeast A kinases have specific signaling functions in pseudohyphal growth. Proc. Natl. Acad. Sci. U.S.A. 95, 13783-13787. doi: 10.1073/pnas.95.23.13783

Sambrook, J., Fritsch, E. F., and Maniatis, T. (1989). Molecular Cloning. New York, NY: Cold spring harbor laboratory press.

Saunders, D. G., Aves, S. J., and Talbot, N. J. (2010). Cell cycle-mediated regulation of plant infection by the rice blast fungus. Plant Cell 22, 497-507. doi: 10.1105/ tpc.109.072447

Schumacher, J., Kokkelink, L., Huesmann, C., Jimenez-Teja, D., Collado, I. G., Barakat, R., et al. (2008). The cAMP-dependent signaling pathway and its role in conidial germination, growth, and virulence of the gray mold Botrytis cinerea. Mol. Plant Microbe Interact. 21, 1443-1459. doi: 10.1094/MPMI-21-111443

Selvaraj, P., Hong Fai, T., Ramanujam, R., and Naqvi, N. I. (2017). Subcellular compartmentation, interdependency and dynamics of the cyclic AMPdependent PKA subunits during pathogenic differentiation in Rice Blast. Mol. Microbiol. 105, 484-504. doi: 10.1111/mmi.13713

Smith, F. D., and Scott, J. D. (2006). Anchored cAMP signaling: onward and upward-a short history of compartmentalized cAMP signal transduction. Eur. J. Cell Biol. 85, 585-592. doi: 10.1016/j.ejcb.2006.01.011

Sonneborn, A., Bockmühl, D. P., Gerads, M., Kurpanek, K., Sanglard, D., and Ernst, J. F. (2000). Protein kinase A encoded by TPK2 regulates dimorphism of Candida albicans. Mol. Microbiol. 35, 386-396. doi: 10.1046/j.1365-2958.2000. 01705.x

Takano, Y., Komeda, K., Kojima, K., and Okuno, T. (2001). Proper regulation of cyclic AMP-dependent protein kinase is required for growth, conidiation, and appressorium function in the anthracnose fungus Colletotrichum lagenarium. Mol. Plant Microbe Interact. 14, 1149-1157. doi: 10.1094/MPMI.2001.14.10. 1149

Thompson, J. D., Higgins, D. G., and Gibson, T. J. (1994). CLUSTAL W: improving the sensitivity of progressive multiple sequence alignment through sequence weighting, position-specific gap penalties and weight matrix choice. Nucleic Acids Res. 22, 4673-4680. doi: 10.1093/nar/22.22.4673

Toda, T., Cameron, S., Sass, P., Zoller, M., and Wigler, M. (1987). Three different genes in $S$. cerevisiae encode the catalytic subunits of the cAMPdependent protein kinase. Cell 50, 277-287. doi: 10.1016/0092-8674(87) 90223-6

Tzima, A., Paplomatas, E. J., Rauyaree, P., and Kang, S. (2010). Roles of the catalytic subunit of cAMP-dependent protein kinase A in virulence and development of the soilborne plant pathogen Verticillium dahliae. Fungal Genet. Biol. 47, 406-415. doi: 10.1016/j.fgb.2010.01.007

Veneault-Fourrey, C., Barooah, M., Egan, M., Wakley, G., and Talbot, N. J. (2006). Autophagic fungal cell death is necessary for infection by the rice blast fungus. Science 312, 580-583. doi: 10.1126/science.1124550

Xu, J.-R., and Hamer, J. E. (1996). MAP kinase and cAMP signaling regulate infection structure formation and pathogenic growth in the rice blast fungus Magnaporthe grisea. Genes Dev. 10, 2696-2706. doi: 10.1101/gad.10.21.2696

Xu, J.-R., Urban, M., Sweigard, J. A., and Hamer, J. E. (1997). The CPKA gene of Magnaporthe grisea is essential for appressorial penetration. Mol. Plant Microbe Interact. 10, 187-194. doi: 10.1094/MPMI.1997.10.2.187

Yamauchi, J., Takayanagi, N., Komeda, K., Takano, Y., and Okuno, T. (2004). cAMP-PKA signaling regulates multiple steps of fungal infection cooperatively with Cmk1 MAP kinase in Colletotrichum lagenarium. Mol. Plant Microbe Interact. 17, 1355-1365. doi: 10.1094/MPMI.2004.17.12.1355

Yan, X., and Talbot, N. J. (2016). Investigating the cell biology of plant infection by the rice blast fungus Magnaporthe oryzae. Curr. Opin. Microbiol. 34, 147-153. doi: 10.1016/j.mib.2016.10.001

Yang, F., and Naqvi, N. I. (2014). Sulfonylurea resistance reconstitution as a novel strategy for ILV2-specific integration in Magnaporthe oryzae. Fungal Genet. Biol. 68, 71-76. doi: 10.1016/j.fgb.2014.04.005

Yang, Z., and Dickman, M. B. (1999). Colletotrichum trifolii mutants disrupted in the catalytic subunit of cAMP-dependent protein kinase are nonpathogenic. 
Mol. Plant Microbe Interact. 12, 430-439. doi: 10.1094/MPMI.1999.12. 5.430

Zhang, H., Liu, K., Zhang, X., Tang, W., Wang, J., Guo, M., et al. (2011). Two phosphodiesterase genes, PDEL and PDEH, regulate development and pathogenicity by modulating intracellular cyclic AMP levels in Magnaporthe oryzae. PLOS ONE 6:e17241. doi: 10.1371/journal.pone. 0017241

Zhao, X., Kim, Y., Park, G., and Xu, J.-R. (2005). A mitogen-activated protein kinase cascade regulating infection-related morphogenesis in Magnaporthe grisea. Plant Cell 17, 1317-1329. doi: 10.1105/tpc.104.029116
Conflict of Interest Statement: The authors declare that the research was conducted in the absence of any commercial or financial relationships that could be construed as a potential conflict of interest.

Copyright (c) 2017 Selvaraj, Shen, Yang and Naqvi. This is an open-access article distributed under the terms of the Creative Commons Attribution License (CC BY). The use, distribution or reproduction in other forums is permitted, provided the original author(s) or licensor are credited and that the original publication in this journal is cited, in accordance with accepted academic practice. No use, distribution or reproduction is permitted which does not comply with these terms. 